Homology, Homotopy and Applications, vol.7(1), 2005, pp.9-37

\title{
PRODUCT STRUCTURES ON FOUR DIMENSIONAL SOLVABLE LIE ALGEBRAS
}

\author{
A. ANDRADA, M. L. BARBERIS, I. G. DOtTI AND G. P. OVANDO \\ (communicated by Charles Weibel)
}

\begin{abstract}
It is the aim of this work to study product structures on four dimensional solvable Lie algebras. We determine all possible paracomplex structures and consider the case when one of the subalgebras is an ideal. These results are applied to the case of Manin triples and complex product structures. We also analyze the three dimensional subalgebras.
\end{abstract}

\section{Introduction}

A product structure on a smooth manifold $M$ is an endomorphism $E$ of its tangent bundle satisfying $E^{2}=\mathrm{Id}$ together with

$E[X, Y]=[E X, Y]+[X, E Y]-E[E X, E Y]$ for all vector fields $X, Y$ on $M$. (1)

A product structure on $M$ gives rise to a splitting of the tangent bundle $T M$ into the Whitney sum of two subbundles $T^{ \pm} M$ corresponding to the \pm 1 eigenspaces of $E$. The distributions on $M$ defined by $T^{+} M$ and $T^{-} M$ are completely integrable. When $T^{+} M$ and $T^{-} M$ have the same rank the product structure is called a paracomplex structure.

Product structures on manifolds were considered by many authors from different points of view. Examples of Riemannian almost product structures were given in [Miq] and a survey on paracomplex geometry can be found in [CFG]. The classification of Riemannian almost product manifolds according to a certain decomposition of the space of tensors was done in $[\mathbf{N}]$. In $[\mathbf{L M}]$ the authors give a new look at singular and non holonomic Lagrangian systems in the framework of almost product structures. Complex product structures on Lie groups were considered in $[\mathbf{A S}]$ and [BV].

In this paper we consider product structures on four dimensional solvable Lie groups. Such groups provide an important source of applications in geometry. Invariant structures on the group, for instance, special metrics $[\mathbf{A l}],[\mathbf{B 2}],[\mathbf{D S}],[\mathbf{F 1}]$, $[\mathbf{F 2}],[\mathbf{J}]$, complex and Kähler structures $[\mathbf{A C F M}],[\mathbf{A F G M}],[\mathbf{O 1}],[\mathbf{S J}],[\mathbf{F G}]$, hypercomplex and hypersymplectic structures $[\mathbf{A n}],[\mathbf{B} \mathbf{1}]$, can be read off in $\mathbb{R}^{4}$,

The authors were partially supported by CONICET and SECYT-UNC (Argentina).

Received December 30, 2003, revised December 28, 2004; published on April 7, 2005.

2000 Mathematics Subject Classification: Primary 53C15; Secondary 22E25.

Key words and phrases: solvable Lie algebra, product structure, paracomplex structure.

(C) 2005, A. Andrada, M. L. Barberis, I. G. Dotti and G. P. Ovando. Permission to copy for private use granted. 
the universal covering group, giving often explicit descriptions of the corresponding structure.

A left-invariant product structure on a Lie group is determined by its restriction to the corresponding Lie algebra, considered as the tangent space at the identity. A product structure on a Lie algebra $\mathfrak{g}$ is a linear endomorphism $E: \mathfrak{g} \longrightarrow \mathfrak{g}$ satisfying $E^{2}=\mathrm{Id}$ (and not equal to $\pm \mathrm{Id}$ ) and

$$
E[x, y]=[E x, y]+[x, E y]-E[E x, E y] \quad \text { for all } x, y \in \mathfrak{g} .
$$

A product structure on $\mathfrak{g}$ gives rise to a decomposition of $\mathfrak{g}$ into

$$
\mathfrak{g}=\mathfrak{g}_{+} \oplus \mathfrak{g}_{-}, \quad E\left|\mathfrak{g}_{+}=\mathrm{Id}, E\right| \mathfrak{g}_{-}=-\mathrm{Id},
$$

where both, $\mathfrak{g}_{+}$and $\mathfrak{g}_{-}$, are Lie subalgebras of $\mathfrak{g}$. This will be denoted $\mathfrak{g}=\mathfrak{g}_{+} \bowtie \mathfrak{g}_{-}$, since the structure of $\mathfrak{g}$ is that of a double Lie algebra ([LW]). In case both $\mathfrak{g}_{+}$and $\mathfrak{g}$ - have the same dimension we say that $\mathfrak{g}$ carries a paracomplex structure.

The outline of this paper is as follows. In Section 1 we describe all non-isomorphic four dimensional solvable Lie algebras over $\mathbb{R}$. This was studied by Mubarakzyanov $[\mathbf{M u}]$ and Dozias $[\mathbf{D}]$. We found citations of the theorems obtained by Mubarakzyanov in [PSWZ], pp. 988 and Dozias in $[\mathbf{V e}]$, pp. 180. We include a proof of the classification theorem since it will be frequently used to obtain the results throughout the article. Appendix II contains comparisons with the tables given by the various authors $[\mathbf{M u}],[\mathbf{D}],[\mathbf{S J}],[\mathbf{O 1}],[\mathbf{P S W Z}]$.

In Section 2 we consider product structures on four dimensional Lie algebras. We determine all four dimensional solvable Lie algebras admitting a paracomplex structure (see Table 2). Among these, we study the case when one of the subalgebras is an ideal of $\mathfrak{g}$. We also exhibit decompositions where one of the subalgebras is three dimensional (see Table 3).

An important subclass of paracomplex structures is given by Manin triples and complex product structures (see Section 3). A paracomplex structure $\mathfrak{g}=\mathfrak{g}_{+} \bowtie \mathfrak{g}_{-}$ is a Manin triple if there exists a non degenerate invariant symmetric bilinear form on $\mathfrak{g}$ such that $\mathfrak{g}_{ \pm}$are isotropic subalgebras. It is shown that there is only one non abelian four dimensional solvable Lie algebra giving rise to a Manin triple. On the other hand, given a product structure $E$ and a complex structure $J$ on $\mathfrak{g}$ such that $J E=-E J,\{J, E\}$ is called a complex product structure on $\mathfrak{g}$. We determine all four dimensional solvable Lie algebras admitting complex product structures (see Table 4), giving an alternative proof of a result by Blazić and Vukmirović ([BV]).

\section{Classification of four dimensional solvable Lie algebras}

In this section we obtain the classification of four dimensional solvable Lie algebras. The proof follows the lines of [Mi] for the classification of three dimensional solvable Lie algebras, that is, we obtain the four dimensional solvable Lie algebras as extensions of the three dimensional unimodular Lie algebras $\mathbb{R}^{3}$, the Heisenberg algebra $\mathfrak{h}_{3}$, the Poincaré algebra $\mathfrak{e}(1,1)$ or the Euclidean algebra $\mathfrak{e}(2)$. Both, $[\mathbf{O 1}]$ and $[\mathbf{S J}]$, obtain the four dimensional solvable Lie algebras as extensions of nilpotent Lie algebras of dimension at most three. In Appendix I we exhibit matrix realizations 
and Appendix II contains comparisons with the tables given by the various authors $[\mathbf{M u}],[\mathbf{D}],[\mathbf{S J}],[\mathbf{O 1}],[\mathbf{P S W Z}]$.

\subsection{Algebraic preliminaries}

A Lie algebra $\mathfrak{g}$ which satisfies the condition $\operatorname{tr}(\operatorname{ad}(x))=0$ for all $x \in \mathfrak{g}$ will be called a unimodular Lie algebra. If $\mathfrak{g}$ is a Lie algebra, then using the Jacobi identity we see that $\operatorname{tr}(\operatorname{ad}[x, y])=0$ for all $x, y \in \mathfrak{g}$. Hence, the map $\chi: \mathfrak{g} \rightarrow \mathbb{R}$ defined by

$$
\chi(x)=\operatorname{tr}(\operatorname{ad}(x)), \quad x \in \mathfrak{g},
$$

is a Lie algebra homomorphism. In particular, its kernel $\mathfrak{u}=\operatorname{ker}(\chi)$ is an ideal containing the commutator ideal $[\mathfrak{g}, \mathfrak{g}]$. The ideal $\mathfrak{u}$ will be called the unimodular kernel of $\mathfrak{g}$. It is easy to check that $\mathfrak{u}$ itself is unimodular.

We now introduce some notation that will be used throughout the paper (compare with $[\mathbf{G O V}])$.

$\mathfrak{a f f}(\mathbb{R}):\left[e_{1}, e_{2}\right]=e_{2}$, the two dimensional non-abelian Lie algebra of the group of affine motions of the real line;

$\mathfrak{h}_{3}: \quad\left[e_{1}, e_{2}\right]=e_{3}$, the three-dimensional Heisenberg algebra;

$\mathfrak{r}_{3}: \quad\left[e_{1}, e_{2}\right]=e_{2},\left[e_{1}, e_{3}\right]=e_{2}+e_{3}$

$\mathfrak{r}_{3, \lambda}: \quad\left[e_{1}, e_{2}\right]=e_{2},\left[e_{1}, e_{3}\right]=\lambda e_{3}$

$\mathfrak{r}_{3, \lambda}^{\prime}: \quad\left[e_{1}, e_{2}\right]=\lambda e_{2}-e_{3},\left[e_{1}, e_{3}\right]=e_{2}+\lambda e_{3} ;$

Remark. Observe that $\mathfrak{r}_{3,-1}$ is the Lie algebra $\mathfrak{e}(1,1)$ of the group of rigid motions of Minkowski 2-space, $\mathfrak{r}_{3,0}=\mathbb{R} \times \mathfrak{a f f}(\mathbb{R})$ and $\mathfrak{r}_{3,1}$ is the Lie algebra of the solvable group which acts simply and transitively on the real hyperbolic space $\mathbb{R} H^{3}$. Also $\mathfrak{r}_{3,0}^{\prime}$ is the Lie algebra $\mathfrak{e}(2)$ of the group of rigid motions of Euclidean 2-space. Other authors denote $\mathfrak{a f f}(\mathbb{R})$ by $\mathfrak{s o l}_{2}$ and $\mathfrak{e}(1,1)$ by $\mathfrak{s o l}_{3}$.

We recall the classification of solvable Lie algebras of dimension $\leqslant 3$. A proof can be found, for example, in $[\mathbf{M i}]$ or $[\mathbf{G O V}]$.

Theorem 1.1. Let $\mathfrak{g}$ be a real solvable Lie algebra, $\operatorname{dim} \mathfrak{g} \leqslant 3$. Then $\mathfrak{g}$ is isomorphic to one and only one of the following Lie algebras: $\mathbb{R}, \mathbb{R}^{2}, \mathfrak{a f f}(\mathbb{R}), \mathbb{R}^{3}, \mathfrak{h}_{3}, \mathfrak{r}_{3}$, $\mathfrak{r}_{3, \lambda},|\lambda| \leqslant 1$ and $\mathfrak{r}_{3, \lambda}^{\prime}, \lambda \geqslant 0$. Among these, the unimodular ones are $\mathbb{R}, \mathbb{R}^{2}, \mathbb{R}^{3}$, $\mathfrak{h}_{3}, \mathfrak{r}_{3,-1}$, and $\mathfrak{r}_{3,0}^{\prime}$.

The proof of Theorem 1.5 in next section is based on the knowledge of the algebra of derivations of solvable unimodular three dimensional Lie algebras. This is the content of the next lemma, whose proof is straightforward.

Lemma 1.2. The algebra of derivations of $\mathfrak{e}(2), \mathfrak{e}(1,1)$ and $\mathfrak{h}_{3}$ are

$$
\operatorname{Der} \mathfrak{e}(2)=\left\{\left(\begin{array}{ccc}
0 & 0 & 0 \\
c & a & -b \\
d & b & a
\end{array}\right): a, b, c, d \in \mathbb{R}\right\},
$$

with respect to the basis $e_{i}, i=1,2,3$, such that $\left[e_{1}, e_{2}\right]=e_{3}, \quad\left[e_{1}, e_{3}\right]=-e_{2}$;

$$
\operatorname{Der} \mathfrak{e}(1,1)=\left\{\left(\begin{array}{lll}
0 & 0 & 0 \\
c & a & 0 \\
d & 0 & b
\end{array}\right): a, b, c, d \in \mathbb{R}\right\} \cong \mathfrak{a} \mathfrak{f}(\mathbb{R}) \times \mathfrak{a f f}(\mathbb{R}),
$$


with respect to the basis $e_{i}, i=1,2,3$ such that $\left[e_{1}, e_{2}\right]=e_{2}, \quad\left[e_{1}, e_{3}\right]=-e_{3}$;

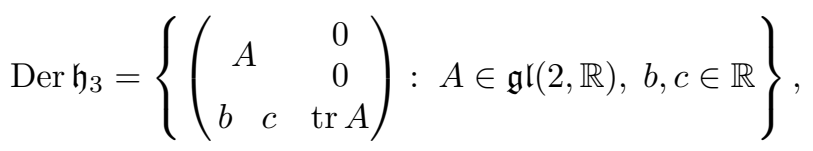

with respect to the basis $e_{i}, \quad i=1,2,3$ such that $\left[e_{1}, e_{2}\right]=e_{3}$.

\subsection{Classification theorem}

In this section we obtain all four dimensional solvable Lie algebras as semidirect extensions of three dimensional unimodular Lie algebras. The classification theorem is then reduced to the study of the derivations of these three dimensional algebras. The proof will follow the lines of $[\mathbf{M i}]$ for the three dimensional case, but instead of the rational form, we make use of the Jordan normal form over $\mathbb{R}$.

Given a Lie algebra $\mathfrak{g}$ and an ideal $\mathfrak{v}$ of codimension one in $\mathfrak{g}$, let $e_{0} \in \mathfrak{g} \backslash \mathfrak{v}$. Then we denote

$$
\mathfrak{g}=\mathbb{R} e_{0} \ltimes_{\varphi} \mathfrak{v},
$$

where $\varphi: \mathbb{R} e_{0} \rightarrow$ Der $\mathfrak{v}$ is a linear map such that $\varphi\left(e_{0}\right)=\operatorname{ad}\left(e_{0}\right)$. Observe that the splitting of the short exact sequence

$$
0 \rightarrow \mathfrak{v} \rightarrow \mathfrak{g} \rightarrow \mathbb{R} \rightarrow 0,
$$

is an immediate consequence of the fact that $\mathbb{R}$ is one dimensional.

The following result proves the desired decomposition, that is, any four dimensional solvable real Lie algebra is a semidirect product of $\mathbb{R}$ and a three-dimensional unimodular ideal. Thus this proposition is a first step in the classification (compare with Proposition 2.1 in $[\mathbf{D S}])$ :

Proposition 1.3. Let $\mathfrak{g}$ be a four-dimensional solvable real Lie algebra. Then there is a short exact sequence

$$
0 \rightarrow \mathfrak{v} \rightarrow \mathfrak{g} \rightarrow \mathbb{R} \rightarrow 0,
$$

where $\mathfrak{v}$ is an ideal of $\mathfrak{g}$ isomorphic to either $\mathbb{R}^{3}, \mathfrak{h}_{3}, \mathfrak{e}(1,1)$ or $\mathfrak{e}(2)$, that is, $\mathfrak{g} \cong$ $\mathbb{R} e_{0} \ltimes_{\varphi} \mathfrak{v}$.

Proof. Consider the Lie algebra homomorphism $\chi: \mathfrak{g} \rightarrow \mathbb{R}$ defined in (4). If $\mathfrak{g}$ is not unimodular then its unimodular kernel $\mathfrak{u}$ has dimension three, therefore it is isomorphic to $\mathbb{R}^{3}, \mathfrak{h}_{3}, \mathfrak{e}(1,1)$ or $\mathfrak{e}(2)$ and the proposition follows with $\mathfrak{v}=\mathfrak{u}$.

We assume now that $\mathfrak{g}$ is unimodular. The commutator ideal $\mathfrak{g}^{\prime}$ is nilpotent and $\operatorname{dim} \mathfrak{g}^{\prime} \leqslant 3$, hence it follows that $\mathfrak{g}^{\prime}$ is isomorphic to $\{0\}, \mathbb{R}, \mathbb{R}^{2}, \mathbb{R}^{3}$ or $\mathfrak{h}_{3}$. In the last two cases the proposition follows by taking $\mathfrak{v}=\mathfrak{g}^{\prime}$. If $\mathfrak{g}^{\prime}=\{0\}$ then $\mathfrak{g}$ is abelian so that $\mathfrak{v}=\mathbb{R}^{3}$ is an ideal of $\mathfrak{g}$.

If $\mathfrak{g}^{\prime}$ is isomorphic to $\mathbb{R}, \mathfrak{g}^{\prime}=\mathbb{R} e_{3}$, then there exist elements $e_{1}, e_{2}$ in $\mathfrak{g}$ such that $\left[e_{1}, e_{2}\right]=e_{3}$. The set $e_{1}, e_{2}, e_{3}$ is linearly independent since $\mathfrak{g}$ is unimodular. Therefore, the Lie subalgebra generated by $e_{1}, e_{2}, e_{3}$ is an ideal isomorphic to $\mathfrak{h}_{3}$.

If $\mathfrak{g}^{\prime}$ is isomorphic to $\mathbb{R}^{2}$ then either i) there exists $x$ not in $\mathfrak{g}^{\prime}$ such that $\operatorname{ad}(x)_{\mid \mathfrak{g}^{\prime}}$ is non singular, or ii) for all $x \in \mathfrak{g}$ the transformation $\operatorname{ad}(x)$ is singular. Making use of the Jordan form of the corresponding complex transformation we get in both 
cases i) and ii), that $\chi(x)=\lambda_{1}+\lambda_{2}=0$, for $\lambda_{i} \in \mathbb{C}, i=1$ or 2 . Thus in case i) there is a basis of $\mathfrak{g}^{\prime}$ such that the action of $x$ is given as follows (up to a nonzero multiple):
a) $\left.\operatorname{ad}(x)\right|_{\mathfrak{g}^{\prime}}=\left(\begin{array}{cc}1 & 0 \\ 0 & -1\end{array}\right)$
or
b) $\left.\operatorname{ad}(x)\right|_{\mathfrak{g}^{\prime}}=\left(\begin{array}{cc}0 & 1 \\ -1 & 0\end{array}\right)$

where case b) corresponds to the eigenvalues $i,-i$. Thus $\mathbb{R} x \oplus \mathfrak{g}^{\prime}$ is an ideal of $\mathfrak{g}$ isomorphic to $\mathfrak{e}(1,1)$ or to $\mathfrak{e}(2)$, respectively.

In case ii), since $\lambda_{1}$ or $\lambda_{2}$ is zero, then the unimodular condition imposes that both eigenvalues vanish and so, for a fixed $x$ not in $\mathfrak{g}^{\prime}$, there is a basis of $\mathfrak{g}^{\prime}$ such that the action of $\left.\operatorname{ad}(x)\right|_{\mathfrak{g}^{\prime}}$ takes one of the following forms:

$$
\text { a) }\left.\operatorname{ad}(x)\right|_{\mathfrak{g}^{\prime}}=0 \quad \text { or } \quad \text { b) }\left.\operatorname{ad}(x)\right|_{\mathfrak{g}^{\prime}}=\left(\begin{array}{ll}
0 & 1 \\
0 & 0
\end{array}\right)
$$

Therefore, $\mathbb{R} x \oplus \mathfrak{g}^{\prime}$ is an ideal of $\mathfrak{g}$ isomorphic to $\mathbb{R}^{3}$ in case a) or $\mathfrak{h}_{3}$ in case b). This completes the proof.

The following lemma will be used in the proof of the classification theorem.

Lemma 1.4. Let $\mathfrak{g}_{1}=\mathbb{R} e_{0} \ltimes_{\varphi_{1}} \mathbb{R}^{3}$ and $\mathfrak{g}_{2}=\mathbb{R} e_{0} \ltimes_{\varphi_{2}} \mathbb{R}^{3}$ such that $\left[\mathfrak{g}_{i}, \mathfrak{g}_{i}\right]=\mathbb{R}^{3}$, $i=$ 1,2 . Then $\mathfrak{g}_{1} \cong \mathfrak{g}_{2}$ if and only if there exists $\gamma \neq 0$ such that $\varphi_{1}\left(e_{0}\right)$ and $\gamma \varphi_{2}\left(e_{0}\right)$ are conjugate in $G L(3, \mathbb{R})$.

Proof. Assume first that there exists a Lie algebra isomorphism $\psi: \mathfrak{g}_{1} \rightarrow \mathfrak{g}_{2}$; then $\psi: \mathbb{R}^{3} \rightarrow \mathbb{R}^{3}$ and $\psi\left(e_{0}\right)=\gamma e_{0}+w$, where $\gamma \in \mathbb{R} \backslash\{0\}$ and $w \in \mathbb{R}^{3}$. If $v \in \mathbb{R}^{3}$, we calculate

$$
\begin{aligned}
{\left[\psi\left(e_{0}\right), \psi(v)\right] } & =\gamma \varphi_{2}\left(e_{0}\right) \psi(v), \\
\psi\left(\left[e_{0}, v\right]\right) & =\psi\left(\varphi_{1}\left(e_{0}\right) v\right),
\end{aligned}
$$

and therefore $\gamma \varphi_{2}\left(e_{0}\right) \psi(v)=\psi\left(\varphi_{1}\left(e_{0}\right) v\right)$ for all $v \in \mathbb{R}^{3}$, that is, $\gamma \varphi_{2}\left(e_{0}\right)$ $=\psi \varphi_{1}\left(e_{0}\right) \psi^{-1}$.

The converse is straightforward.

Dozias and Mubarakzyanov gave in $[\mathbf{D}]$ and $[\mathbf{M u}]$ a classification of four dimensional solvable Lie algebras. We prove below this result to make this article self contained. The proof uses Proposition 1.3 together with Lemma 1.2.

Theorem 1.5. Let $\mathfrak{g}$ be a four-dimensional solvable real Lie algebra. Then $\mathfrak{g}$ is isomorphic to one and only one of the following Lie algebras: $\mathbb{R}^{4}, \mathfrak{a f f}(\mathbb{R}) \times \mathfrak{a f f}(\mathbb{R}), \mathbb{R} \times$ $\mathfrak{h}_{3}, \quad \mathbb{R} \times \mathfrak{r}_{3}, \quad \mathbb{R} \times \mathfrak{r}_{3, \lambda}, \quad|\lambda| \leqslant 1, \quad \mathbb{R} \times \mathfrak{r}_{3, \lambda}^{\prime}, \quad \lambda \geqslant 0, \quad$ or one of the Lie algebras with brackets given below in the basis $e_{i}, \quad i=0,1,2,3$ :

$$
\begin{aligned}
\mathfrak{n}_{4}: & {\left[e_{0}, e_{1}\right]=e_{2}, \quad\left[e_{0}, e_{2}\right]=e_{3} ; } \\
\mathfrak{a f f}(\mathbb{C}): & {\left[e_{0}, e_{2}\right]=e_{2}, \quad\left[e_{0}, e_{3}\right]=e_{3}, \quad\left[e_{1}, e_{2}\right]=e_{3}, \quad\left[e_{1}, e_{3}\right]=-e_{2} ; } \\
\mathfrak{r}_{4}: & {\left[e_{0}, e_{1}\right]=e_{1}, \quad\left[e_{0}, e_{2}\right]=e_{1}+e_{2}, \quad\left[e_{0}, e_{3}\right]=e_{2}+e_{3} } \\
\mathfrak{r}_{4, \lambda}: & {\left[e_{0}, e_{1}\right]=e_{1}, \quad\left[e_{0}, e_{2}\right]=\lambda e_{2}, \quad\left[e_{0}, e_{3}\right]=e_{2}+\lambda e_{3} }
\end{aligned}
$$




$$
\begin{aligned}
\mathfrak{r}_{4, \mu, \lambda}: & {\left[e_{0}, e_{1}\right]=e_{1}, \quad\left[e_{0}, e_{2}\right]=\mu e_{2}, \quad\left[e_{0}, e_{3}\right]=\lambda e_{3}, \quad \mu \lambda \neq 0, \quad-1<\mu \leqslant \lambda \leqslant 1 } \\
\text { or } & -1=\mu \leqslant \lambda<0 ; \\
\mathfrak{r}_{4, \mu, \lambda}^{\prime}: & {\left[e_{0}, e_{1}\right]=\mu e_{1}, \quad\left[e_{0}, e_{2}\right]=\lambda e_{2}-e_{3}, \quad\left[e_{0}, e_{3}\right]=e_{2}+\lambda e_{3}, \quad \mu>0 ; } \\
\mathfrak{d}_{4}: & {\left[e_{0}, e_{1}\right]=e_{1}, \quad\left[e_{0}, e_{2}\right]=-e_{2}, \quad\left[e_{1}, e_{2}\right]=e_{3} ; } \\
\mathfrak{d}_{4, \lambda}: & {\left[e_{0}, e_{1}\right]=\lambda e_{1}, \quad\left[e_{0}, e_{2}\right]=(1-\lambda) e_{2}, \quad\left[e_{0}, e_{3}\right]=e_{3}, \quad\left[e_{1}, e_{2}\right]=e_{3}, \quad \lambda \geqslant \frac{1}{2} ; } \\
\mathfrak{d}_{4, \lambda}^{\prime}: & {\left[e_{0}, e_{1}\right]=\lambda e_{1}-e_{2}, \quad\left[e_{0}, e_{2}\right]=e_{1}+\lambda e_{2}, \quad\left[e_{0}, e_{3}\right]=2 \lambda e_{3}, \quad\left[e_{1}, e_{2}\right]=} \\
e_{3}, & \lambda \geqslant 0 ; \\
\mathfrak{h}_{4}: & {\left[e_{0}, e_{1}\right]=e_{1},\left[e_{0}, e_{2}\right]=e_{1}+e_{2},\left[e_{0}, e_{3}\right]=2 e_{3}, \quad\left[e_{1}, e_{2}\right]=e_{3} . }
\end{aligned}
$$

Among these, the unimodular algebras are: $\mathbb{R}^{4}, \quad \mathbb{R} \times \mathfrak{h}_{3}, \quad \mathbb{R} \times \mathfrak{r}_{3,-1}, \quad \mathbb{R} \times$ $\mathfrak{r}_{3,0}^{\prime}, \quad \mathfrak{n}_{4}, \quad \mathfrak{r}_{4,-1 / 2}, \mathfrak{r}_{4, \mu,-1-\mu}(-1<\mu \leqslant-1 / 2), \quad \mathfrak{r}_{4, \mu,-\mu / 2}^{\prime}, \quad \mathfrak{d}_{4}, \quad \mathfrak{d}_{4,0}^{\prime}$.

Proof. In view of Proposition 1.3 there exists a three dimensional ideal $\mathfrak{v}$ of $\mathfrak{g}$ isomorphic to $\mathbb{R}^{3}, \mathfrak{e}(2), \mathfrak{e}(1,1)$ or $\mathfrak{h}_{3}$. We will analyze below the different cases.

\subsection{Case $\mathfrak{v}=\mathbb{R}^{3}$.}

We introduce first the following $3 \times 3$ real matrices which will be needed in the next paragraphs:

$$
\begin{aligned}
A_{1}^{\mu, \lambda} & =\left(\begin{array}{lll}
1 & 0 & 0 \\
0 & \mu & 0 \\
0 & 0 & \lambda
\end{array}\right), & A_{2}^{\lambda} & =\left(\begin{array}{ccc}
1 & 0 & 0 \\
0 & \lambda & 1 \\
0 & 0 & \lambda
\end{array}\right), \\
A_{3} & =\left(\begin{array}{lll}
1 & 1 & 0 \\
0 & 1 & 1 \\
0 & 0 & 1
\end{array}\right), & A_{4}^{\mu, \lambda} & =\left(\begin{array}{ccc}
\mu & 0 & 0 \\
0 & \lambda & 1 \\
0 & -1 & \lambda
\end{array}\right) .
\end{aligned}
$$

By assumption, $\mathfrak{g}=\mathbb{R} e_{0} \ltimes_{\varphi} \mathbb{R}^{3}$ where $\varphi\left(e_{0}\right)=\operatorname{ad}\left(e_{0}\right)$. Suppose first that $\varphi\left(e_{0}\right)$ has real eigenvalues. We have the following possibilities for $\varphi\left(e_{0}\right)$, where the eigenvalues are ordered such that $\left|\lambda_{1}\right| \leqslant\left|\lambda_{2}\right| \leqslant\left|\lambda_{3}\right|$ :

$$
\begin{gathered}
\text { i) } \varphi\left(e_{0}\right)=\left(\begin{array}{ccc}
\lambda_{1} & 0 & 0 \\
0 & \lambda_{2} & 0 \\
0 & 0 & \lambda_{3}
\end{array}\right), \quad \text { ii) } \varphi\left(e_{0}\right)=\left(\begin{array}{ccc}
\lambda_{1} & 0 & 0 \\
0 & \lambda_{2} & 1 \\
0 & 0 & \lambda_{2}
\end{array}\right), \\
\text { iii) } \varphi\left(e_{0}\right)=\left(\begin{array}{ccc}
\lambda & 1 & 0 \\
0 & \lambda & 1 \\
0 & 0 & \lambda
\end{array}\right) . \\
\text { Case i) } \begin{cases}\lambda_{i}=0, i=1,2,3, & \text { then } \mathfrak{g} \cong \mathbb{R}^{4} ; \\
\lambda_{1}=0, \lambda_{3} \neq 0, & \text { then } \mathfrak{g} \cong \mathfrak{r}_{3, \lambda} \times \mathbb{R} ; \text { where } \lambda=\frac{\lambda_{2}}{\lambda_{3}} ; \\
\lambda_{1} \lambda_{2} \lambda_{3} \neq 0 & \text { then } \mathfrak{g} \cong \mathbb{R} \ltimes_{\varphi_{1}} \mathbb{R}^{3}, \varphi_{1}\left(e_{0}\right)=A_{1}^{\mu, \lambda} \text { as shown in }(9),\end{cases}
\end{gathered}
$$

The last isomorphism in Case i) follows by dividing $e_{0}$ by $\lambda_{3}$ and by reordering suitably the basis $\left\{e_{1}, e_{2}, e_{3}\right\}$ of $\mathbb{R}^{3}$, we may assume that $-1 \leqslant \mu \leqslant \lambda \leqslant 1$. 
Case ii) $\begin{cases}\lambda_{1}=\lambda_{2}=0 & \text { then } \mathfrak{g} \cong \mathbb{R} \times \mathfrak{h}_{3} ; \\ \lambda_{1}=0, \lambda_{2} \neq 0 & \text { then } \mathfrak{g} \cong \mathbb{R} \times \mathfrak{r}_{3} ; \\ \lambda_{1} \neq 0, & \text { then } \mathfrak{g} \cong \mathbb{R} \ltimes_{\varphi_{2}^{\lambda}} \mathbb{R}^{3}, \quad \varphi_{2}^{\lambda}\left(e_{0}\right)=A_{2}^{\lambda} \text { as shown in (9), } \\ & \text { that is, } \mathfrak{g} \cong \mathfrak{r}_{4, \lambda} .\end{cases}$

Case iii) $\begin{cases}\lambda=0, & \text { then } \mathfrak{g} \cong \mathfrak{n}_{4} ; \\ \lambda \neq 0, & \text { then } \mathfrak{g} \cong \mathbb{R} \ltimes_{\varphi_{3}} \mathbb{R}^{3}, \varphi_{3}\left(e_{0}\right)=A_{3} \text { as shown in (10), that is, } \\ & \mathfrak{g} \cong \mathfrak{r}_{4} .\end{cases}$

The last isomorphism in case iii) follows by taking $e_{0} / \lambda$.

In case $\varphi\left(e_{0}\right)$ has only one real eigenvalue, $\mu$, then we may assume that $\varphi\left(e_{0}\right)=A_{4}^{\mu, \lambda}$ as in (10) and we have:

$$
\left\{\begin{array}{l}
\mu=0, \text { then } \mathfrak{g} \cong \mathbb{R} \times \mathfrak{r}_{3, \lambda}^{\prime} \\
\mu \neq 0, \text { then } \mathfrak{g} \cong \mathfrak{r}_{4, \mu, \lambda}^{\prime}, \mu>0
\end{array}\right.
$$

Observe that the last isomorphism follows by changing $e_{0}$ by $-e_{0}$.

1.4. Case $\mathfrak{v}=\mathfrak{e}(2)$.

Assume that $\mathfrak{g}=\mathbb{R} e_{0} \ltimes_{\varphi} \mathfrak{e}(2)$ where $\varphi\left(e_{0}\right)=\operatorname{ad}\left(e_{0}\right) \in \operatorname{Der} \mathfrak{e}(2)$ is as in (5). Then setting $e_{0}^{\prime}=e_{0}-b e_{1}+d e_{2}-c e_{3}$, it follows that

$$
\left[e_{0}^{\prime}, e_{1}\right]=0, \quad\left[e_{0}^{\prime}, e_{2}\right]=a e_{2}, \quad\left[e_{0}^{\prime}, e_{3}\right]=a e_{3} ;
$$

therefore, $\mathfrak{g} \cong \mathbb{R} \times \mathfrak{e}(2)=\mathbb{R} \times \mathfrak{r}_{3,0}^{\prime}$ or $\mathfrak{g} \cong \mathfrak{a} \mathfrak{f} f(\mathbb{C})$ depending on $a=0$ or $a \neq 0$, respectively.

1.5. Case $\mathfrak{v}=\mathfrak{e}(1,1)$.

Assume that $\mathfrak{g}=\mathbb{R} e_{0} \ltimes_{\varphi} \mathfrak{e}(1,1)$ where $\varphi\left(e_{0}\right)=\operatorname{ad}\left(e_{0}\right) \in \operatorname{Der} \mathfrak{e}(1,1)$ is as in (6). Let $e_{0}^{\prime}=e_{0}-a e_{1}+c e_{2}-d e_{3}$, then

$$
\left[e_{0}^{\prime}, e_{1}\right]=0, \quad\left[e_{0}^{\prime}, e_{2}\right]=0, \quad\left[e_{0}^{\prime}, e_{3}\right]=(a+b) e_{3} ;
$$

therefore, $\mathfrak{g} \cong \mathbb{R} \times \mathfrak{e}(1,1)=\mathbb{R} \times \mathfrak{r}_{3,-1}$ or $\mathfrak{g} \cong \mathfrak{a f f}(\mathbb{R}) \times \mathfrak{a} \mathfrak{f}(\mathbb{R})$ depending on $a+b=0$ or $a+b \neq 0$, respectively.

1.6. Case $\mathfrak{v}=\mathfrak{h}_{3}$.

Assume that $\mathfrak{g} \cong \mathbb{R} e_{0} \ltimes_{\varphi} \mathfrak{h}_{3}$ where $\varphi\left(e_{0}\right)=\operatorname{ad}\left(e_{0}\right)$ is given by

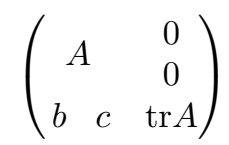

(see (7)). We may assume that $b=c=0$. In fact, setting $e_{0}^{\prime}=e_{0}-c e_{1}+b e_{2}$ it turns out that $\operatorname{ad}\left(e_{0}^{\prime}\right)$ is given by

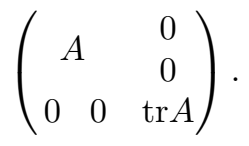


Assume first that $A$ has two real eigenvalues $\gamma, \beta$; then $A$ takes the form
i) $A=\left(\begin{array}{ll}\gamma & 0 \\ 0 & \beta\end{array}\right)$,
or
ii) $A=\left(\begin{array}{ll}\gamma & 1 \\ 0 & \gamma\end{array}\right)$.

Observe that in all cases, once we change the basis $e_{1}, e_{2}$ to $e_{1}^{\prime}, e_{2}^{\prime}$, we must set $e_{3}^{\prime}=\left[e_{1}^{\prime}, e_{2}^{\prime}\right]$ in order to obtain a Lie algebra isomorphism.

$$
\text { Case i) }\left\{\begin{array} { l } 
{ \gamma = \beta = 0 , \text { then } \mathfrak { g } \cong \mathbb { R } \times \mathfrak { h } _ { 3 } ; } \\
{ \gamma = - \beta \neq 0 , \text { then } \mathfrak { g } \cong \mathfrak { d } _ { 4 } ; } \\
{ \gamma + \beta \neq 0 , \text { then } \mathfrak { g } \cong \mathfrak { d } _ { 4 , \lambda } , \quad \lambda = \frac { \gamma } { \gamma + \beta } ; }
\end{array} \quad \text { Case ii) } \left\{\begin{array}{l}
\gamma=0, \text { then } \mathfrak{g} \cong \mathfrak{n}_{4} ; \\
\gamma \neq 0, \text { then } \mathfrak{g} \cong \mathfrak{h}_{4} .
\end{array}\right.\right.
$$

We show that $\mathfrak{d}_{4, \lambda} \cong \mathfrak{d}_{4,1-\lambda}$. This follows by changing the basis $e_{i}, 0 \leqslant i \leqslant 3$, to the basis $e_{i}^{\prime}, 0 \leqslant i \leqslant 3$, where:

$$
e_{0}^{\prime}=e_{0}, \quad e_{1}^{\prime}=e_{2}, \quad e_{2}^{\prime}=e_{1}, \quad e_{3}^{\prime}=-e_{3} .
$$

Therefore, we may assume that $\lambda \geqslant 1 / 2$.

In case ii), $\gamma \neq 0$, in order to show that $\mathfrak{g} \cong \mathfrak{h}_{4}$ one has to start with $e_{0}^{\prime}=\frac{1}{\gamma} e_{0}$, then take $e_{1}^{\prime}, e_{2}^{\prime} \in \operatorname{span}\left\{e_{1}, e_{2}\right\}$ such that

$$
\operatorname{ad}\left(e_{0}^{\prime}\right)=\left(\begin{array}{ll}
1 & 1 \\
0 & 1
\end{array}\right)
$$

with respect to $\left\{e_{1}^{\prime}, e_{2}^{\prime}\right\}$ and $e_{3}^{\prime}=\left[e_{1}^{\prime}, e_{2}^{\prime}\right]$.

If $A$ has no real eigenvalues, then $\operatorname{ad}\left(e_{0}^{\prime}\right)$ takes the form

$$
\left(\begin{array}{ccc}
\lambda & 1 & 0 \\
-1 & \lambda & 0 \\
0 & 0 & 2 \lambda
\end{array}\right)
$$

and we conclude that $\mathfrak{g} \cong \mathfrak{d}_{4, \lambda}^{\prime}$. Hence, we have shown so far that any four dimensional solvable Lie algebra is isomorphic to one of those listed in the statement of the theorem. It remains to show that they are pairwise non isomorphic.

\subsection{Isomorphism classes}

In Table 1, we list the four dimensional solvable Lie algebras according to their commutator. After that, we proceed to distinguish them up to isomorphism.

- $[\mathfrak{g}, \mathfrak{g}]=\mathbb{R}: \mathbb{R} \times \mathfrak{h}_{3}$ is nilpotent but $\mathbb{R} \times \mathfrak{r}_{3,0}$ is not, therefore they are not isomorphic.

- $[\mathfrak{g}, \mathfrak{g}]=\mathbb{R}^{2}, \mathfrak{z}=\{0\}:$ Both $\mathfrak{a} \mathfrak{f} \mathfrak{f}(\mathbb{R}) \times \mathfrak{a f f}(\mathbb{R})$ and $\mathfrak{d}_{4,1}$ are completely solvable ${ }^{1}$ and therefore not isomorphic to $\mathfrak{a f f}(\mathbb{C})$, which is not completely solvable. The unimodular kernel of $\mathfrak{a f f}(\mathbb{R}) \times \mathfrak{a f f}(\mathbb{R})\left(\right.$ resp. $\left.\mathfrak{d}_{4,1}\right)$ is $\mathfrak{r}_{3,-1}\left(\right.$ resp. $\left.\mathfrak{h}_{3}\right)$, hence $\mathfrak{a f f}(\mathbb{R}) \times \mathfrak{a f f}(\mathbb{R})$ is not isomorphic to $\mathfrak{d}_{4,1}$.

${ }^{1}$ Recall that a solvable Lie algebra $\mathfrak{g}$ is completely solvable when ad $(x)$ has real eigenvalues for all $x \in \mathfrak{g}$. 


\begin{tabular}{|l|l|}
\hline \multicolumn{1}{|c|}{$[\mathfrak{g}, \mathfrak{g}]$} & $\mathfrak{g}$ \\
\hline \hline$\{0\}$ & $\mathbb{R}^{4}$ \\
\hline $\mathbb{R}$ & $\mathbb{R} \times \mathfrak{h}_{3}, \quad \mathbb{R} \times \mathfrak{r}_{3,0}$ \\
\hline $\mathbb{R}^{2}, \mathfrak{z}=\{0\}$ & $\mathfrak{a} \mathfrak{f}(\mathbb{R}) \times \mathfrak{a} \mathfrak{f}(\mathbb{R}), \quad \mathfrak{a} \mathfrak{f}(\mathbb{C}), \quad \mathfrak{d}_{4,1}$ \\
\hline $\mathbb{R}^{2}, \mathfrak{z} \neq\{0\}$ & $\mathbb{R} \times \mathfrak{r}_{3}, \quad \mathbb{R} \times \mathfrak{r}_{3, \lambda}(|\lambda| \leqslant 1, \lambda \neq 0), \quad \mathbb{R} \times \mathfrak{r}_{3, \lambda}^{\prime}(\lambda \geqslant 0), \quad \mathfrak{r}_{4,0}, \quad \mathfrak{n}_{4}$ \\
\hline $\mathbb{R}^{3}$ & $\mathfrak{r}_{4}, \quad \mathfrak{r}_{4, \lambda}(\lambda \neq 0), \mathfrak{r}_{4, \mu, \lambda}(\mu \lambda \neq 0,-1 \leqslant \mu \leqslant \lambda \leqslant 1), \mathfrak{r}_{4, \mu, \lambda}^{\prime}(\mu>0)$ \\
\hline $\mathfrak{h}_{3}$ & $\mathfrak{d}_{4}, \mathfrak{d}_{4, \lambda}(\lambda \neq 1, \lambda \geqslant 1 / 2), \quad \mathfrak{d}_{4, \lambda}^{\prime}(\lambda \geqslant 0), \quad \mathfrak{h}_{4}$ \\
\hline
\end{tabular}

Table 1:

$\bullet[\mathfrak{g}, \mathfrak{g}]=\mathbb{R}^{2}, \mathfrak{z} \neq\{0\}:$ If $\mathfrak{g}=\mathbb{R} \times \mathfrak{r}_{3}, \quad \mathbb{R} \times \mathfrak{r}_{3, \lambda}(|\lambda| \leqslant 1, \lambda \neq 0)$ or $\mathbb{R} \times \mathfrak{r}_{3, \lambda}^{\prime}(\lambda \geqslant 0)$ then $\mathfrak{z} \cap[\mathfrak{g}, \mathfrak{g}]=\{0\}$, while $\mathfrak{z} \cap[\mathfrak{g}, \mathfrak{g}] \neq\{0\}$ when $\mathfrak{g}=\mathfrak{r}_{4,0}$ or $\mathfrak{n}_{4}$. Also $\mathfrak{g}=$ $\mathbb{R} \times \mathfrak{r}_{3}, \quad \mathbb{R} \times \mathfrak{r}_{3, \lambda}(|\lambda| \leqslant 1, \lambda \neq 0)$ and $\mathbb{R} \times \mathfrak{r}_{3, \lambda}^{\prime}(\lambda \geqslant 0)$ are not pairwise isomorphic since $\mathfrak{r}_{3}, \mathfrak{r}_{3, \lambda}$ and $\mathfrak{r}_{3, \lambda}^{\prime}$ are 3 -dimensional non isomorphic Lie algebras. On the other hand, $\mathfrak{n}_{4}$ is nilpotent but $\mathfrak{r}_{4,0}$ is not, hence they are not isomorphic.

- $[\mathfrak{g}, \mathfrak{g}]=\mathbb{R}^{3}: \mathfrak{r}_{4}, \mathfrak{r}_{4, \lambda}(\lambda \neq 0), \mathfrak{r}_{4, \mu, \lambda}, \mathfrak{r}_{4, \mu, \lambda}^{\prime}$. In this case, it follows from Lemma 1.4 that any pair of Lie algebras belonging to different families can not be isomorphic. The last family consists of non completely solvable Lie algebras.

The fact that two Lie algebras $\mathfrak{r}_{4, \lambda}, \lambda \neq 0$, and $\mathfrak{r}_{4, \lambda^{\prime}}, \lambda^{\prime} \neq 0$, are isomorphic if and only if $\lambda=\lambda^{\prime}$ follows by applying Lemma 1.4.

Let us show that if $\mathfrak{r}_{4, \mu, \lambda},-1<\mu \leqslant \lambda \leqslant 1, \mu \lambda \neq 0$, is isomorphic to $\mathfrak{r}_{4, \mu^{\prime}, \lambda^{\prime}},-1<\mu^{\prime} \leqslant \lambda^{\prime} \leqslant 1, \mu^{\prime} \lambda^{\prime} \neq 0$, then $\mu=\mu^{\prime}$ and $\lambda=\lambda^{\prime}$. From Lemma 1.4 , there exists $\gamma \neq 0$ such that the sets of eigenvalues $\{1, \mu, \lambda\}$ and $\left\{\gamma, \gamma \mu^{\prime}, \gamma \lambda^{\prime}\right\}$ must coincide. If $\gamma=1$ the desired assertion follows from $\mu \leqslant \lambda$ and $\mu^{\prime} \leqslant \lambda^{\prime}$. If $\gamma=\mu$ then either $\gamma \mu^{\prime}=1$ or $\gamma \lambda^{\prime}=1$, hence $\mu^{\prime}=1$ or $\lambda^{\prime}=1$, therefore $\gamma=1$ and again this implies $\mu=\mu^{\prime}, \quad \lambda=\lambda^{\prime}$. The case $\gamma=\lambda$ is proved in a similar way.

Let us show that if $\mathfrak{r}_{4,-1, \lambda},-1 \leqslant \lambda<0$, is isomorphic to $\mathfrak{r}_{4,-1, \lambda^{\prime}},-1 \leqslant \lambda^{\prime}<0$, then $\lambda=\lambda^{\prime}$. We apply Lemma 1.4 again to obtain that there exists $\gamma \neq 0$ such that $\{1,-1, \lambda\}$ and $\left\{\gamma,-\gamma, \gamma \lambda^{\prime}\right\}$ must coincide. We cannot have $\gamma=-1$, since this would imply $\lambda=-\lambda^{\prime}$, a contradiction, since both, $\lambda$ and $\lambda^{\prime}$ are negative. If $\gamma=\lambda$, then $-\gamma=1$ and $-1=\gamma \lambda^{\prime}=\lambda \lambda^{\prime}>0$, a contradiction. Thus $\gamma=1$ and $\lambda=\lambda^{\prime}$.

If $\mathfrak{r}_{4, \mu, \lambda},-1<\mu \leqslant \lambda \leqslant 1, \mu \lambda \neq 0$, were isomorphic to $\mathfrak{r}_{4,-1, \lambda^{\prime}},-1 \leqslant \lambda^{\prime}<0$, then Lemma 1.4 would imply that that there exists $\gamma \neq 0$ such that $\{1, \mu, \lambda\}=$ $\left\{\gamma,-\gamma, \gamma \lambda^{\prime}\right\}$. If $\gamma=1$ then $\mu=-1$, which is impossible. On the other hand, $\gamma=\mu$ implies $-\gamma=1$ or $\gamma \lambda^{\prime}=1$, hence $\mu=\gamma=-1$, a contradiction. The case $\gamma=\lambda$ is similar; therefore, the above Lie algebras are not isomorphic.

Assume now that $\mathfrak{r}_{4, \mu, \lambda}^{\prime}, \mu>0$, is isomorphic to $\mathfrak{r}_{4, \mu^{\prime}, \lambda^{\prime}}^{\prime}, \mu^{\prime}>0$, we must show that $\mu=\mu^{\prime}$ and $\lambda=\lambda^{\prime}$. We apply Lemma 1.4 again to obtain that there exists $\gamma \neq 0$ such that $\mu=\gamma \mu^{\prime}$ and $\lambda \pm i=\gamma\left(\lambda^{\prime} \pm i\right)$. It follows from the second equality that $\gamma= \pm 1$, and the first equality implies $\gamma=1$, since both $\mu$ and $\mu^{\prime}$ are positive. Therefore, $\mu=\mu^{\prime}$ and $\lambda=\lambda^{\prime}$, as claimed.

- $[\mathfrak{g}, \mathfrak{g}]=\mathfrak{h}_{3}$ : The Lie algebras $\mathfrak{d}_{4}, \mathfrak{d}_{4, \lambda}(\lambda \geqslant 1 / 2, \lambda \neq 1)$, and $\mathfrak{d}_{4, \lambda}^{\prime}, \mathfrak{h}_{4}$ are 
distinguished by $\mathfrak{g} / \mathfrak{z}([\mathfrak{g}, \mathfrak{g}])$, as the following table shows:

\begin{tabular}{|c||c|c|c|c|}
\hline $\mathfrak{g}$ & $\mathfrak{d}_{4}$ & $\mathfrak{d}_{4, \lambda}, \begin{array}{l}\lambda \geqslant 1 / 2 \\
\lambda \neq 1\end{array}$ & $\mathfrak{d}_{4, \lambda}^{\prime}, \quad \lambda \geqslant 0$ & $\mathfrak{h}_{4}$ \\
\hline $\mathfrak{g} / \mathfrak{z}([\mathfrak{g}, \mathfrak{g}])$ & $\mathfrak{r}_{3,-1}$ & $\mathfrak{r}_{3,-1+1 / \lambda}$ & $\mathfrak{r}_{3, \lambda}^{\prime}$ & $\mathfrak{r}_{3}$ \\
\hline
\end{tabular}

Remarks. (i) In [DS $]$ it was proved that $\mathfrak{d}_{4, \lambda}^{\prime}, \lambda \geqslant 0$, are all non-isomorphic. Observe that $\mathfrak{g}_{\lambda}$ in $[\mathbf{D S}]$ corresponds to $\mathfrak{d}_{4,1 / \lambda}^{\prime}$ for $\lambda \neq 0$ (resp. $\mathfrak{d}_{4,1 / 2}$ for $\lambda=0$ ).

(ii) We observe that $\mathfrak{a f f}(\mathbb{C})$ is the Lie algebra of the group of affine motions of the complex line, which is isomorphic to the complexification of $\mathfrak{a f f}(\mathbb{R})$ looked upon as a real Lie algebra. [It should be noted that the Lie algebra Der $\mathfrak{e}(2)$ (see Lemma 1.2 ) is isomorphic to $\mathfrak{a f f}(\mathbb{C})$.] Also, $\mathfrak{r}_{4,1,1}$ is the Lie algebra of a solvable Lie group which acts simply and transitively on the real hyperbolic space $\mathbb{R} H^{4}$ and $\mathfrak{d}_{4,1 / 2}$ is the Lie algebra of a solvable Lie group which acts simply and transitively on the complex hyperbolic space $\mathbb{C} H^{2}$.

\section{Product structures on four dimensional solvable Lie alge- bras}

\subsection{Basic definitions}

An almost product structure on a Lie algebra $\mathfrak{g}$ is a linear endomorphism $E$ : $\mathfrak{g} \longrightarrow \mathfrak{g}$ satisfying $E^{2}=\mathrm{Id}$ (and not equal to $\pm \mathrm{Id}$ ). It is said to be integrable if

$$
E[x, y]=[E x, y]+[x, E y]-E[E x, E y] \quad \text { for all } x, y \in \mathfrak{g} .
$$

An integrable almost product structure will be called a product structure.

An almost product structure on $\mathfrak{g}$ gives rise to a decomposition of $\mathfrak{g}$ into

$$
\mathfrak{g}=\mathfrak{g}_{+} \oplus \mathfrak{g}_{-}, \quad E\left|\mathfrak{g}_{+}=\mathrm{Id}, E\right| \mathfrak{g}_{-}=-\mathrm{Id} .
$$

The integrability of $E$ is equivalent to $\mathfrak{g}_{+}$and $\mathfrak{g}_{-}$being subalgebras. When $\operatorname{dim} \mathfrak{g}_{+}=$ $\operatorname{dim} \mathfrak{g}_{-}$, the product structure $E$ is called a paracomplex structure.

Three Lie algebras $\left(\mathfrak{g}, \mathfrak{g}_{+}, \mathfrak{g}_{-}\right)$form a double Lie algebra if $\mathfrak{g}_{+}$and $\mathfrak{g}_{-}$are Lie subalgebras of $\mathfrak{g}$ and $\mathfrak{g}=\mathfrak{g}_{+} \oplus \mathfrak{g}_{-}$as vector spaces. This will be denoted by $\mathfrak{g}=$ $\mathfrak{g}_{+} \bowtie \mathfrak{g}_{-}$. Observe that a double Lie algebra $\left(\mathfrak{g}, \mathfrak{g}_{+}, \mathfrak{g}_{-}\right)$gives a product structure $E: \mathfrak{g} \longrightarrow \mathfrak{g}$ on $\mathfrak{g}$, where $E \mid \mathfrak{g}_{+}=$Id and $E \mid \mathfrak{g}_{-}=-$Id. Conversely, a product structure on the Lie algebra $\mathfrak{g}$ gives rise to a double Lie algebra $\left(\mathfrak{g}, \mathfrak{g}_{+}, \mathfrak{g}_{-}\right)$, where $\mathfrak{g}_{ \pm}$is the eigenspace associated to the eigenvalue \pm 1 of $E$. The notion of double Lie algebra is a natural generalization of that of semidirect product. We will denote $\mathfrak{g}=\mathfrak{g}_{+} \ltimes \mathfrak{g}_{-}$ the semidirect product of $\mathfrak{g}_{+}$and $\mathfrak{g}_{-}$where $\mathfrak{g}_{-}$is an ideal of $\mathfrak{g}$, that is, there is a split exact sequence

$$
0 \longrightarrow \mathfrak{g}_{-} \longrightarrow \mathfrak{g} \longrightarrow \mathfrak{g}_{+} \longrightarrow 0
$$

Product structures or, equivalently, double Lie algebras, were used in several contexts (see $[\mathbf{A S}],[\mathbf{L W}]$ ). Important examples of double Lie algebras are Manin triples and complex product structures. 


\begin{tabular}{|c|c|c|c|}
\hline $\mathfrak{g}$ & $\mathbb{R}^{2} \bowtie \mathbb{R}^{2}$ & $\mathfrak{a f f f}(\mathbb{R}) \bowtie \mathbb{R}^{2}$ & $\mathfrak{a} \mathfrak{a} \mathfrak{f}(\mathbb{R}) \bowtie \mathfrak{a} \mathfrak{f} \mathfrak{f}(\mathbb{R})$ \\
\hline $\mathbb{R}^{4}$ & $\left\langle e_{0}, e_{1}\right\rangle \times\left\langle e_{2}, e_{3}\right\rangle$ & no & no \\
\hline $\mathfrak{a f f f}(\mathbb{R}) \times \mathfrak{a f f}(\mathbb{R})$ & $\left\langle e_{0}, e_{1}\right\rangle \ltimes\left\langle e_{2}, e_{3}\right\rangle$ & $\begin{array}{c}\left\langle e_{1}+e_{3}, e_{2}\right\rangle \\
\bowtie\left\langle e_{0}, e_{1}\right\rangle\end{array}$ & $\left\langle e_{0}, e_{3}\right\rangle \times\left\langle e_{1}, e_{2}\right\rangle$ \\
\hline $\mathbb{R} \times \mathfrak{h}_{3}$ & $\left\langle e_{0}, e_{2}\right\rangle \ltimes\left\langle e_{1}, e_{3}\right\rangle$ & no & no \\
\hline $\mathbb{R} \times \mathfrak{r}_{3}$ & $\left\langle e_{0}, e_{1}\right\rangle \ltimes\left\langle e_{2}, e_{3}\right\rangle$ & $\left\langle e_{1}, e_{2}\right\rangle \bowtie\left\langle e_{0}, e_{3}\right\rangle$ & no \\
\hline $\mathbb{R} \times \mathfrak{r}_{3, \lambda}, \lambda \neq 0$ & $\left\langle e_{0}, e_{1}\right\rangle \ltimes\left\langle e_{2}, e_{3}\right\rangle$ & $\left\langle e_{1}, e_{2}\right\rangle \ltimes\left\langle e_{0}, e_{3}\right\rangle$ & $\begin{array}{c}\left\langle e_{0}+e_{1}, e_{2}\right\rangle \\
\bowtie\left\langle e_{1}-\lambda e_{0}, e_{3}\right\rangle\end{array}$ \\
\hline $\mathbb{R} \times \mathfrak{r}_{3,0}$ & $\left\langle e_{0}, e_{1}\right\rangle \ltimes\left\langle e_{2}, e_{3}\right\rangle$ & $\left\langle e_{1}, e_{2}\right\rangle \times\left\langle e_{0}, e_{3}\right\rangle$ & no \\
\hline $\mathbb{R} \times \mathfrak{r}_{3, \lambda}^{\prime}$ & $\left\langle e_{0}, e_{1}\right\rangle \ltimes\left\langle e_{2}, e_{3}\right\rangle$ & no & no \\
\hline $\mathfrak{n}_{4}$ & $\left\langle e_{0}, e_{3}\right\rangle \bowtie\left\langle e_{1}, e_{2}\right\rangle$ & no & no \\
\hline $\mathfrak{a f f}(\mathbb{C})$ & $\left\langle e_{0}, e_{1}\right\rangle \ltimes\left\langle e_{2}, e_{3}\right\rangle$ & $\begin{array}{c}\left\langle e_{0}, e_{2}\right\rangle \\
\bowtie\left\langle e_{0}-e_{3}, e_{1}+e_{2}\right\rangle\end{array}$ & no \\
\hline $\mathfrak{r}_{4}$ & no & $\left\langle e_{0}, e_{1}\right\rangle \bowtie\left\langle e_{2}, e_{3}\right\rangle$ & no \\
\hline $\mathfrak{r}_{4, \lambda}, \lambda \neq 0$ & no & $\left\langle e_{0}, e_{1}\right\rangle \ltimes\left\langle e_{2}, e_{3}\right\rangle$ & $\begin{array}{c}\left\langle e_{0}, e_{1}\right\rangle \\
\bowtie\left\langle e_{0}+\lambda e_{3}, e_{2}\right\rangle\end{array}$ \\
\hline $\mathfrak{r}_{4,0}$ & $\left\langle e_{0}, e_{2}\right\rangle \bowtie\left\langle e_{1}, e_{3}\right\rangle$ & $\left\langle e_{0}, e_{1}\right\rangle \ltimes\left\langle e_{2}, e_{3}\right\rangle$ & no \\
\hline $\mathfrak{r}_{4, \mu, \lambda}$ & no & $\left\langle e_{0}, e_{1}\right\rangle \ltimes\left\langle e_{2}, e_{3}\right\rangle$ & $\begin{array}{c}\left\langle e_{0}-e_{1}, e_{2}\right\rangle \\
\bowtie\left\langle e_{0}+e_{1}, e_{3}\right\rangle\end{array}$ \\
\hline $\mathfrak{r}_{4, \mu, \lambda}^{\prime}$ & no & $\left\langle e_{0}, e_{1}\right\rangle \ltimes\left\langle e_{2}, e_{3}\right\rangle$ & no \\
\hline $\mathfrak{d}_{4}$ & no & $\left\langle e_{0}, e_{1}\right\rangle \ltimes\left\langle e_{2}, e_{3}\right\rangle$ & $\begin{array}{c}\left\langle e_{0}+e_{2}, e_{1}-e_{3}\right\rangle \\
\bowtie\left\langle e_{0}-e_{2}, e_{1}+e_{3}\right\rangle\end{array}$ \\
\hline $\mathfrak{d}_{4, \lambda}, \lambda \neq 1$ & no & $\left\langle e_{0}, e_{1}\right\rangle \ltimes\left\langle e_{2}, e_{3}\right\rangle$ & $\begin{array}{c}\left\langle e_{0}, e_{3}\right\rangle \bowtie\left\langle e_{0}+\lambda e_{2},\right. \\
\left.(1-\lambda) e_{1}+\lambda e_{3}\right\rangle\end{array}$ \\
\hline $\mathfrak{d}_{4,1}$ & $\left\langle e_{0}, e_{2}\right\rangle \ltimes\left\langle e_{1}, e_{3}\right\rangle$ & $\left\langle e_{0}, e_{1}\right\rangle \ltimes\left\langle e_{2}, e_{3}\right\rangle$ & $\begin{array}{c}\left\langle e_{0}, e_{1}\right\rangle \\
\bowtie\left\langle e_{0}+e_{2}, e_{3}\right\rangle\end{array}$ \\
\hline $\mathfrak{d}_{4, \lambda}^{\prime}$ & no & no & no \\
\hline $\mathfrak{h}_{4}$ & no & $\left\langle e_{0}, e_{1}\right\rangle \bowtie\left\langle e_{2}, e_{3}\right\rangle$ & $\begin{array}{c}\left\langle e_{0}, e_{3}\right\rangle \\
\bowtie\left\langle e_{0}-e_{2}, e_{1}-e_{3}\right\rangle\end{array}$ \\
\hline
\end{tabular}

Table 2: Paracomplex structures on four dimensional solvable Lie algebras

\subsection{Paracomplex structures}

It is the main goal of this subsection to determine all 4-dimensional solvable Lie algebras admitting paracomplex structures. We will give realizations of the Lie algebras obtained in Theorem 1.5 as double Lie algebras with subalgebras of dimension 2 when such a structure exists (see Table 2), or prove the non existence otherwise. It turns out that among all four dimensional solvable Lie algebras there is only one family, whose commutator ideal is $\mathfrak{h}_{3}$, not admitting any paracomplex structure (Theorem 2.7). Since there are only two non-isomorphic two-dimensional Lie algebras: $\mathbb{R}^{2}$ and $\mathfrak{a f f}(\mathbb{R})$, the possible decompositions $\mathfrak{g}_{+} \bowtie \mathfrak{g}_{-}$are $\mathbb{R}^{2} \bowtie \mathbb{R}^{2}, \mathbb{R}^{2} \bowtie \mathfrak{a f f}(\mathbb{R})$ and $\mathfrak{a} \mathfrak{f} \mathfrak{f}(\mathbb{R}) \bowtie \mathfrak{a f f}(\mathbb{R})$.

By simple computations one can verify that the decompositions given in Table 2 satisfy the required properties. We prove below the non existence results.

Proposition 2.1. Let $\mathfrak{g}$ be a Lie algebra with an abelian commutator ideal $\mathfrak{g}^{\prime}$ of codimension 1 . Then any abelian subalgebra of dimension $n>1$ is contained in $\mathfrak{g}^{\prime}$. 
Proof. In this case there is $e_{0} \in \mathfrak{g}$ such that $\operatorname{ad}\left(e_{0}\right)$ is an isomorphism of $\mathfrak{g}^{\prime}$. Let $\mathfrak{h}$ be an abelian subalgebra of $\mathfrak{g}, \operatorname{dim} \mathfrak{h}>1$, and let $x, y \in \mathfrak{h}$ linearly independent. If $x=a_{0} e_{0}+x^{\prime}, y=b_{0} e_{0}+y^{\prime}$ with $a_{0}, b_{0} \in \mathbb{R}$ and $x^{\prime}, y^{\prime} \in \mathfrak{g}^{\prime}$, then

$$
0=[x, y]=\left[e_{0}, a_{0} y^{\prime}-b_{0} x^{\prime}\right]
$$

This implies that $a_{0} y^{\prime}-b_{0} x^{\prime}=0$, that is, $a_{0} y-b_{0} x=0$ and hence $a_{0}=b_{0}=0$. Therefore, $x, y \in \mathfrak{g}^{\prime}$, as asserted.

The previous result together with Table 1 imply

Corollary 2.2. The Lie algebras $\mathfrak{r}_{4}, \mathfrak{r}_{4, \lambda}(\lambda \neq 0), \mathfrak{r}_{4, \mu, \lambda}, \mathfrak{r}_{4, \mu, \lambda}^{\prime}$ do not admit a decomposition of type $\mathbb{R}^{2} \bowtie \mathbb{R}^{2}$.

Proposition 2.3 ([P]). If $\mathfrak{g}$ is a Lie algebra which admits a decomposition $\mathfrak{g}=$ $\mathfrak{g}_{+} \bowtie \mathfrak{g}_{-}$with $\mathfrak{g}_{+}$and $\mathfrak{g}_{-}$abelian subalgebras, then $\mathfrak{g}$ is 2-step solvable (i.e., $\mathfrak{g}^{\prime}$ is abelian).

Proof. If $\mathfrak{g}=\mathfrak{g}_{+} \bowtie \mathfrak{g}_{-}$with $\mathfrak{g}_{+}$and $\mathfrak{g}_{-}$abelian then $\left[\left(x_{1}, x_{2}\right),\left(y_{1}, y_{2}\right)\right]$ is determined by $\left[\left(x_{1}, 0\right),\left(0, y_{2}\right)\right]=\left(\alpha\left(x_{1}, y_{2}\right), \beta\left(x_{1}, y_{2}\right)\right)$ where $\alpha$ and $\beta$ denote the components on $\mathfrak{g}_{+}$and $\mathfrak{g}_{-}$respectively. Since the bracket on $\mathfrak{g}$ satisfies the Jacobi identity one obtains

1. $\alpha\left(x_{1}, \beta\left(y_{1}, z_{2}\right)\right)=\alpha\left(y_{1}, \beta\left(x_{1}, z_{2}\right)\right)$,

2. $\beta\left(\alpha\left(z_{1}, y_{2}\right), x_{2}\right)=\beta\left(\alpha\left(z_{1}, x_{2}\right), y_{2}\right)$,

3. $\beta\left(x_{1}, \beta\left(y_{1}, z_{2}\right)\right)=\beta\left(y_{1}, \beta\left(x_{1}, z_{2}\right)\right)$,

4. $\left.\left.\alpha\left(\alpha\left(z_{1}, y_{2}\right), x_{2}\right)\right)=\alpha\left(\alpha\left(z_{1}, x_{2}\right), y_{2}\right)\right)$.

Now, using the above relations one can show that

$$
\alpha\left(\alpha\left(x_{1}, y_{2}\right), \beta\left(u_{1}, v_{2}\right)\right)=\alpha\left(\alpha\left(u_{1}, v_{2}\right), \beta\left(x_{1}, y_{2}\right)\right)
$$

and

$$
\beta\left(\alpha\left(x_{1}, y_{2}\right), \beta\left(u_{1}, v_{2}\right)\right)=\beta\left(\alpha\left(u_{1}, v_{2}\right), \beta\left(x_{1}, y_{2}\right)\right) .
$$

But the above relations immediately imply

$$
\left[\left[\left(x_{1}, 0\right),\left(0, y_{2}\right)\right],\left[\left(u_{1}, 0\right),\left(0, v_{2}\right)\right]\right]=0
$$

and the assertion follows.

The above proposition together with Table 1 imply

Corollary 2.4. The Lie algebras $\mathfrak{d}_{4}, \mathfrak{d}_{4, \lambda}(\lambda \neq 1), \mathfrak{d}_{4, \lambda}^{\prime}, \mathfrak{h}_{4}$ do not admit a decomposition of type $\mathbb{R}^{2} \bowtie \mathbb{R}^{2}$.

Lemma 2.5. The Lie algebras $\mathbb{R}^{4}, \mathbb{R} \times \mathfrak{h}_{3}, \mathfrak{n}_{4}$ and $\mathbb{R} \times \mathfrak{r}_{3, \lambda}^{\prime}$ do not contain $\mathfrak{a f f}(\mathbb{R})$ as a subalgebra. Hence, these Lie algebras do not admit decompositions of type $\mathfrak{a} \mathfrak{f} \mathfrak{f}(\mathbb{R}) \bowtie$ $\mathbb{R}^{2}$ or $\mathfrak{a f f}(\mathbb{R}) \bowtie \mathfrak{a f f}(\mathbb{R})$. 
Proof. Since $\mathbb{R}^{4}, \mathbb{R} \times \mathfrak{h}_{3}$ and $\mathfrak{n}_{4}$ are nilpotent, they cannot have subalgebras isomorphic to $\mathfrak{a f f}(\mathbb{R})$. Let us show next that the same holds for $\mathfrak{g}:=\mathbb{R} \times \mathfrak{r}_{3, \lambda}^{\prime}$. In fact, assume that there exist $x, y \in \mathfrak{g}$ such that $[x, y]=y$. Then $y \in \mathfrak{g}^{\prime}$. If $x \in\left\langle e_{0}, e_{2}, e_{3}\right\rangle$ then $y=0$, thus assume that $x=e_{1}+u$. So $[x, y]=\left[e_{1}, y\right]=y$ implies that $y=0$ since $\operatorname{ad}\left(e_{1}\right)$ has no real eigenvalues in $\mathfrak{g}^{\prime}$.

Proposition 2.6. The Lie algebras $\mathbb{R} \times \mathfrak{r}_{3}, \mathbb{R} \times \mathfrak{r}_{3,0}$, aff $(\mathbb{C}), \mathfrak{r}_{4}, \mathfrak{r}_{4,0}$ and $\mathfrak{r}_{4, \mu, \lambda}^{\prime}$ do not admit a decomposition of type $\mathfrak{a f f}(\mathbb{R}) \bowtie \mathfrak{a f f}(\mathbb{R})$.

Proof. Let $\mathfrak{g}:=\mathbb{R} \times \mathfrak{r}_{3}$ and $\mathfrak{h}$ a subalgebra of $\mathfrak{g}$ isomorphic to $\mathfrak{a f f}(\mathbb{R})$. Then $\mathfrak{h}$ has a basis of the form $\left\{e_{1}+u, e_{2}\right\}$ with $u \in\left\langle e_{0}, e_{3}\right\rangle$. Thus, any decomposition of $\mathbb{R} \times \mathfrak{r}_{3}$ of the form $\mathfrak{h}_{1}+\mathfrak{h}_{2}$ with $\mathfrak{h}_{1} \simeq \mathfrak{a f f} \mathfrak{f}(\mathbb{R}) \simeq \mathfrak{h}_{2}$ is not direct since $e_{2} \in \mathfrak{h}_{1} \cap \mathfrak{h}_{2}$. If $\mathfrak{g}=\mathbb{R} \times \mathfrak{r}_{3,0}$, then $\operatorname{dim} \mathfrak{g}^{\prime}=1$ and therefore the assertion follows.

Assume next that $\mathfrak{g} \cong \mathfrak{a f f}(\mathbb{C})$. Every subalgebra of $\mathfrak{g}$ isomorphic to $\mathfrak{a} \mathfrak{f}(\mathbb{R})$ is of the form $\left\langle e_{0}+u, v\right\rangle$ with $u, v \in\left\langle e_{2}, e_{3}\right\rangle$, thus it is contained in the subspace spanned by $\left\{e_{0}, e_{2}, e_{3}\right\}$. Therefore, a aff $(\mathbb{C})$ is not of type $\mathfrak{a} \mathfrak{f} \mathfrak{f}(\mathbb{R}) \bowtie \mathfrak{a f f}(\mathbb{R})$.

If $\mathfrak{g}$ is either $\mathfrak{r}_{4}, \mathfrak{r}_{4,0}$ or $\mathfrak{r}_{4, \mu, \lambda}^{\prime}$, one can show that $e_{1} \in \mathfrak{g}$ belongs to any Lie subalgebra of $\mathfrak{g}$ isomorphic to $\mathfrak{a} \mathfrak{f}(\mathbb{R})$ and thus $\mathfrak{g}$ cannot be decomposed as $\mathfrak{a f f}(\mathbb{R}) \bowtie$ $\mathfrak{a f f}(\mathbb{R})$. We give a proof of this fact in the case $\mathfrak{g}=\mathfrak{r}_{4}$. Let $\mathfrak{u}=\langle u, v\rangle$ be a Lie subalgebra of $\mathfrak{r}_{4}$ isomorphic to $\mathfrak{a f f}(\mathbb{R})$, with $[u, v]=v$. If $u=\sum_{i=0}^{3} a_{i} e_{i}, v=$ $\sum_{i=0}^{3} b_{i} e_{i}$ with $a_{i}, b_{i} \in \mathbb{R}, i=0, \ldots, 3, b_{0}=0$ since $v \in\left[\mathfrak{r}_{4}, \mathfrak{r}_{4}\right]$, then we have

$$
\left\{\begin{array} { l } 
{ a _ { 0 } b _ { 1 } + a _ { 0 } b _ { 2 } = b _ { 1 } , } \\
{ a _ { 0 } b _ { 2 } + a _ { 0 } b _ { 3 } = b _ { 2 } , } \\
{ a _ { 0 } b _ { 3 } = b _ { 3 } }
\end{array} \quad \text { which implies } \left\{\begin{array}{l}
b_{1}\left(a_{0}-1\right)=-a_{0} b_{2}, \\
b_{2}\left(a_{0}-1\right)=-a_{0} b_{3}, \\
b_{3}\left(a_{0}-1\right)=0 .
\end{array}\right.\right.
$$

If $a_{0}-1 \neq 0$, then $b_{3}=0$ and thus $b_{2}\left(a_{0}-1\right)=0$, which implies $b_{2}=0$. From this, we have $b_{1}\left(a_{0}-1\right)=0$, and therefore $b_{1}=0$, i.e. $v=0$, a contradiction. Hence, $a_{0}=1$ and then $b_{2}=b_{3}=0$. Also $b_{1} \in \mathbb{R} \backslash\{0\}$ is arbitrary, and we may take $b_{1}=1$. So,

$$
u=e_{0}+a_{2} e_{2}+a_{3} e_{3}, \quad v=e_{1},
$$

hence, $e_{1} \in \mathfrak{r}_{4}$, as asserted. The proofs of the remaining cases are similar.

Theorem 2.7. If $\mathfrak{g}$ is a four dimensional solvable Lie algebra then $\mathfrak{g}$ does not admit any paracomplex structure if and only if $\mathfrak{g}$ is isomorphic to $\mathfrak{d}_{4, \lambda}^{\prime}$ for some $\lambda \geqslant 0$.

Proof. We first show that if $\mathfrak{g}$ is a Lie algebra in the family $\mathfrak{d}_{4, \lambda}^{\prime}$ with $\lambda \geqslant 0$ then $\mathfrak{g}$ does not admit a paracomplex structure. Let $\mathfrak{u}$ be a 2 -dimensional Lie subalgebra of $\mathfrak{g}$ with a basis $\{u, v\}$, where $u=\sum_{i=0}^{3} a_{i} e_{i}, v=\sum_{i=0}^{3} b_{i} e_{i}$ with $a_{i}, b_{i} \in \mathbb{R}, i=$ $0, \ldots, 3$

Case 1: $[u, v]=0$ and hence $\mathfrak{u} \cong \mathbb{R}^{2}$. In this case we get that

$$
\left\{\begin{array}{l}
\lambda\left(a_{0} b_{1}-a_{1} b_{0}\right)+a_{0} b_{2}-a_{2} b_{0}=0, \\
\lambda\left(a_{0} b_{2}-a_{2} b_{0}\right)-a_{0} b_{1}+a_{1} b_{0}=0, \\
2 \lambda\left(a_{0} b_{3}-a_{3} b_{0}\right)+a_{1} b_{2}-a_{2} b_{1}=0 .
\end{array}\right.
$$


From the first two equations we arrive at $\left(a_{0} b_{2}-a_{2} b_{0}\right)\left(\lambda^{2}+1\right)=0$, and therefore $a_{0} b_{2}-a_{2} b_{0}=0$, which in turn implies $a_{0} b_{1}-a_{1} b_{2}=0$. Summing up, we have

$$
\left(a_{0}, a_{1}, a_{2}\right) \times\left(b_{0}, b_{1}, b_{2}\right)=\left(-2 \lambda\left(a_{0} b_{3}-a_{3} b_{0}\right), 0,0\right)
$$

and hence

$$
\left\{\begin{array}{l}
2 \lambda a_{0}\left(a_{0} b_{3}-a_{3} b_{0}\right)=0, \\
2 \lambda b_{0}\left(a_{0} b_{3}-a_{3} b_{0}\right)=0 .
\end{array}\right.
$$

We have two cases:

(i) $\lambda=0$. Then $\left(a_{0}, a_{1}, a_{2}\right) \times\left(b_{0}, b_{1}, b_{2}\right)=(0,0,0)$ and therefore $\left(b_{0}, b_{1}, b_{2}\right)=$ $\beta\left(a_{0}, a_{1}, a_{2}\right)$, with $\beta \in \mathbb{R}$. Since $u$ and $v$ are linearly independent, we must have $b_{3}-\beta a_{3} \neq 0$. Thus, we obtain that

$$
e_{3}=\frac{1}{b_{3}-\beta a_{3}}(v-\beta u)
$$

(ii) $\lambda \neq 0$. If we suppose $a_{0} b_{3}-a_{3} b_{0} \neq 0$, we arrive at a contradiction; thus $a_{0} b_{3}-$ $a_{3} b_{0}=0$ and $\left(a_{0}, a_{1}, a_{2}\right) \times\left(b_{0}, b_{1}, b_{2}\right)=(0,0,0)$. As in the previous case, we have that $e_{3} \in \mathfrak{u}$.

Case 2: $[u, v]=v$ and hence $\mathfrak{u} \cong \mathfrak{a f f}(\mathbb{R})$. In this case we obtain that $b_{0}=0$ and

$$
\left\{\begin{array}{l}
\lambda a_{0} b_{1}+a_{0} b_{2}=b_{1}, \\
\lambda a_{0} b_{2}-a_{0} b_{1}=b_{2}, \\
2 \lambda a_{0} b_{3}+a_{1} b_{2}-a_{2} b_{1}=b_{3} .
\end{array}\right.
$$

Let us observe first that $a_{0} \neq 0$, since otherwise from (14) we obtain that $b_{1}=b_{2}=$ $b_{3}=0$, i.e. $v=0$, a contradiction. Combining now the first two equations from (14), we arrive at

$$
b_{1} b_{2}\left(\left(\lambda a_{0}-1\right)^{2}+a_{0}^{2}\right)=0 .
$$

Since clearly $\left(\lambda a_{0}-1\right)^{2}+a_{0}^{2} \neq 0$, we have that $b_{1} b_{2}=0$. It is easily seen that this implies $b_{1}=b_{2}=0$. Hence, we need only consider now the equation $2 \lambda a_{0} b_{3}=b_{3}$, with $b_{3} \neq 0$.

(i) $\lambda=0$. In this case, we obtain that $b_{3}=0$, a contradiction. Thus, $\mathfrak{d}_{4,0}^{\prime}$ does not have any Lie subalgebra isomorphic to $\mathfrak{a f f}(\mathbb{R})$.

(ii) $\lambda \neq 0$. Here, since $b_{3} \neq 0$, we have $a_{0}=\frac{1}{2 \lambda}$ and $u$ and $v$ are given by

$$
u=\frac{1}{2 \lambda} e_{0}+a_{1} e_{1}+a_{2} e_{2}, \quad v=e_{3} .
$$

Note that $e_{3} \in \mathfrak{u}$.

In all cases, $e_{3} \in \mathfrak{g}$ belongs to any 2-dimensional Lie subalgebra of $\mathfrak{g}$, and hence this Lie algebra cannot be decomposed as a direct sum (as vector spaces) of two 2-dimensional Lie subalgebras.

The theorem follows by observing that the remaining Lie algebras possess paracomplex structures (see Table 2).

We give next a characterization of the four dimensional solvable Lie algebras which can be decomposed as a semidirect product of two dimensional subalgebras. 


\subsection{Semidirect extensions of $\mathbb{R}^{2}$}

Assume that $\mathfrak{g}$ contains $\mathbb{R}^{2}$ as an ideal and that the short exact sequence

$$
0 \rightarrow \mathbb{R}^{2} \rightarrow \mathfrak{g} \rightarrow \mathfrak{g} / \mathbb{R}^{2} \rightarrow 0
$$

splits. The next result gives a list of the Lie algebras with this property.

Proposition 2.8. Let $\mathfrak{g}$ be a four dimensional solvable Lie algebra.

(i) If there is a split exact sequence

$$
0 \rightarrow \mathbb{R}^{2} \rightarrow \mathfrak{g} \rightarrow \mathbb{R}^{2} \rightarrow 0
$$

then $\mathfrak{g} \cong \mathbb{R}^{4}, \mathfrak{a f f}(\mathbb{R}) \times \mathfrak{a f f}(\mathbb{R}), \mathbb{R} \times \mathfrak{h}_{3}, \mathbb{R} \times \mathfrak{r}_{3}, \mathbb{R} \times \mathfrak{r}_{3, \lambda}, \mathbb{R} \times \mathfrak{r}_{3, \lambda}^{\prime}, \mathfrak{a f f}(\mathbb{C})$ or $\mathfrak{d}_{4,1}$.

(ii) If there is a split exact sequence

$$
0 \rightarrow \mathbb{R}^{2} \rightarrow \mathfrak{g} \rightarrow \mathfrak{a f f}(\mathbb{R}) \rightarrow 0
$$

then $\mathfrak{g} \cong \mathbb{R} \times \mathfrak{r}_{3, \lambda}, \mathfrak{r}_{4, \lambda}, \mathfrak{r}_{4, \mu, \lambda}, \mathfrak{r}_{4, \mu, \lambda}^{\prime}, \mathfrak{d}_{4}$ or $\mathfrak{d}_{4, \lambda}$.

Proof. (i) Table 2 exhibits decompositions of $\mathfrak{g}$ as a semidirect product $\mathbb{R}^{2} \ltimes \mathbb{R}^{2}$ in case $\mathfrak{g} \cong \mathbb{R}^{4}, \mathfrak{a f f}(\mathbb{R}) \times \mathfrak{a f f}(\mathbb{R}), \mathbb{R} \times \mathfrak{h}_{3}, \mathbb{R} \times \mathfrak{r}_{3}, \mathbb{R} \times \mathfrak{r}_{3, \lambda}, \mathbb{R} \times \mathfrak{r}_{3, \lambda}^{\prime}, \mathfrak{a f f f}(\mathbb{C})$ or $\mathfrak{d}_{4,1}$. It follows from Corollaries 2.2 and 2.4 that $\mathfrak{r}_{4}, \mathfrak{r}_{4, \lambda}, \mathfrak{r}_{4, \lambda}^{\prime}, \lambda \neq 0, \mathfrak{r}_{4, \mu, \lambda}, \mathfrak{r}_{4, \mu, \lambda}^{\prime}, \mathfrak{d}_{4}, \mathfrak{d}_{4, \lambda}$, $\lambda \neq 1, \mathfrak{d}_{4, \lambda}^{\prime}$ and $\mathfrak{h}_{4}$ do not admit such a decomposition. It remains to consider the case $\mathfrak{g} \cong \mathfrak{n}_{4}$ or $\mathfrak{r}_{4,0}$. Assume that $\mathfrak{g}=\mathfrak{a} \ltimes \mathfrak{b}$ with $\mathfrak{a} \cong \mathfrak{b} \cong \mathbb{R}^{2}$. Then $\mathfrak{g}^{\prime} \subset \mathfrak{b}$, hence $\mathfrak{g}^{\prime}=\mathfrak{b}$ since in both cases $\mathfrak{g}^{\prime}=\mathbb{R}^{2}$ (Table 1 ).

Consider next the case $\mathfrak{g} \cong \mathfrak{n}_{4}$, hence $\mathfrak{b}=\left\langle e_{2}, e_{3}\right\rangle$ and $\mathfrak{a}=\langle x, y\rangle$ with $x=$ $a e_{0}+b e_{1}+u, y=c e_{0}+d e_{1}+v, a d-b c \neq 0, u, v \in \mathfrak{b}$. We calculate

$$
[x, y]=(a d-b c) e_{2}+\left[e_{0}, a v-c u\right]
$$

which is non zero since the second summand on the right hand side is a multiple of $e_{3}$. This contradicts the fact that $\mathfrak{a} \cong \mathbb{R}^{2}$. Therefore, $\mathfrak{n}_{4}$ does not decompose as $\mathbb{R}^{2} \ltimes \mathbb{R}^{2}$.

The case $\mathfrak{g} \cong \mathfrak{r}_{4,0}$ is similar. We have $\mathfrak{b}=\left\langle e_{1}, e_{2}\right\rangle$ and $\mathfrak{a}=\langle x, y\rangle$ with $x=$ $a e_{0}+b e_{3}+u, y=c e_{0}+d e_{3}+v, a d-b c \neq 0, u, v \in \mathfrak{b}$. We calculate

$$
[x, y]=\left[e_{0}, a v-c u\right]+(a d-b c) e_{2}
$$

which is non zero since the first summand on the right hand side is a multiple of $e_{1}$. This contradicts the fact that $\mathfrak{a} \cong \mathbb{R}^{2}$ and part (i) of the proposition follows.

(ii) If $0 \rightarrow \mathbb{R}^{2} \rightarrow \mathfrak{g} \rightarrow \mathfrak{a f f}(\mathbb{R}) \rightarrow 0$ splits, then there is a subalgebra $\mathfrak{h}$ of $\mathfrak{g}$ isomorphic to $\mathfrak{a f f}(\mathbb{R})$ such that $\mathfrak{g}=\mathfrak{h} \ltimes \mathbb{R}^{2}$. Set

$$
\rho: \mathfrak{h} \rightarrow \mathfrak{g l}(2, \mathbb{R}), \quad \rho(u)=\left.\operatorname{ad}(u)\right|_{\mathbb{R}^{2}}, \quad u \in \mathfrak{h} .
$$

Then $\rho$ is a Lie algebra homomorphism. Let $\mathfrak{h}=\langle x, y\rangle,[x, y]=y$. If $\rho \equiv 0$ then $\mathfrak{g} \cong \mathfrak{a} \mathfrak{f}(\mathbb{R}) \times \mathbb{R}^{2}=\mathbb{R} \times \mathfrak{r}_{3,0}$. If $\operatorname{dim} \operatorname{Im} \rho=1$, then $0=[\rho(x), \rho(y)]=\rho([x, y])=\rho(y)$ and $\rho(x)$ is given as follows:

$$
\left(\begin{array}{cc}
\mu & 0 \\
0 & \lambda
\end{array}\right), \quad \lambda \neq 0, \quad\left(\begin{array}{cc}
\lambda & 1 \\
0 & \lambda
\end{array}\right) \quad \text { or } \quad\left(\begin{array}{cc}
\alpha & \beta \\
-\beta & \alpha
\end{array}\right), \quad \beta \neq 0 .
$$

The first possibility gives $\mathfrak{g} \cong \mathbb{R} \times \mathfrak{r}_{3, \lambda}$ in case $\mu=0$ and $\mathfrak{g} \cong \mathfrak{r}_{4, \mu, \lambda}$ if $\mu \neq 0$. The second possibility yields $\mathfrak{g} \cong \mathfrak{r}_{4, \lambda}$ and the last one gives $\mathfrak{g} \cong \mathfrak{r}_{4,1 / \beta, \alpha / \beta}^{\prime}$. 
If $\operatorname{dim} \operatorname{Im} \rho=2$, then $\rho(x), \rho(y)$ are linearly independent and since $\mathfrak{g}^{\prime}$ is nilpotent and $y \in \mathfrak{g}^{\prime}$, we may assume that

$$
\rho(y)=\left(\begin{array}{ll}
0 & 1 \\
0 & 0
\end{array}\right) .
$$

It follows from $[\rho(x), \rho(y)]=\rho(y)$ that $\rho(x)$ takes the following form:

$$
\rho(x)=\left(\begin{array}{cc}
\alpha+1 / 2 & \beta \\
0 & \alpha-1 / 2
\end{array}\right) .
$$

We can take $\beta=0$ by replacing $x$ with $x-\beta y$. Let us denote by $\mathfrak{g}_{\alpha}$ the Lie algebra corresponding to

$$
\rho(x)=\left(\begin{array}{cc}
\alpha+1 / 2 & 0 \\
0 & \alpha-1 / 2
\end{array}\right), \quad \quad \rho(y)=\left(\begin{array}{ll}
0 & 1 \\
0 & 0
\end{array}\right) .
$$

The following table gives the possibilities for $\mathfrak{g}_{\alpha}$ according to the parameter $\alpha$ :

\begin{tabular}{|c|l|}
\hline$\alpha$ & $\mathfrak{g}_{\alpha}$ \\
\hline$-1 / 2$ & $\mathfrak{d}_{4}$ \\
$1 / 2$ & $\mathfrak{d}_{4,1}$ \\
$\alpha \in(-1 / 2,1 / 2) \cup(1 / 2,3 / 2]$ & $\mathfrak{d}_{4, \lambda}, \lambda=\frac{2}{2 \alpha+1}$ \\
$\alpha \in(-\infty,-1 / 2) \cup(3 / 2, \infty)$ & $\mathfrak{d}_{4, \lambda}, \lambda=\frac{\alpha-1 / 2}{\alpha+1 / 2}$ \\
\hline
\end{tabular}

This completes the proof of the proposition.

\subsection{Semidirect extensions of $\mathfrak{a f f}(\mathbb{R})$}

Assume that $\mathfrak{g}$ contains $\mathfrak{a} \mathfrak{f}(\mathbb{R})$ as an ideal and that the short exact sequence

$$
0 \rightarrow \mathfrak{a f f}(\mathbb{R}) \rightarrow \mathfrak{g} \rightarrow \mathfrak{g} / \mathfrak{a} \mathfrak{f} \mathfrak{f}(\mathbb{R}) \rightarrow 0
$$

splits. The next result states that $\mathfrak{g}$ is a direct product, that is, $\mathfrak{g}$ is isomorphic to $\mathbb{R}^{2} \times \mathfrak{a} \mathfrak{f} f(\mathbb{R})$ or $\mathfrak{a f f}(\mathbb{R}) \times \mathfrak{a f f}(\mathbb{R})$. The precise statement is the following:

Proposition 2.9. Let $\mathfrak{g}$ be a four dimensional solvable Lie algebra.

(i) If there is a split exact sequence

$$
0 \rightarrow \mathfrak{a f f}(\mathbb{R}) \rightarrow \mathfrak{g} \rightarrow \mathbb{R}^{2} \rightarrow 0
$$

then $\mathfrak{g} \cong \mathbb{R} \times \mathfrak{r}_{3,0}=\mathbb{R}^{2} \times \mathfrak{a} \mathfrak{f} \mathfrak{f}(\mathbb{R})$.

(ii) If there is a split exact sequence

$$
0 \rightarrow \mathfrak{a f f}(\mathbb{R}) \rightarrow \mathfrak{g} \rightarrow \mathfrak{a f f}(\mathbb{R}) \rightarrow 0
$$

then $\mathfrak{g} \cong \mathfrak{a f f}(\mathbb{R}) \times \mathfrak{a f f}(\mathbb{R})$.

Proof. Let $\mathfrak{a f f}(\mathbb{R})=\langle z, w\rangle$ with $[z, w]=w$, then the algebra of derivations is given as follows:

$$
\text { Der } \mathfrak{a} \mathfrak{f}(\mathbb{R})=\left\{\left(\begin{array}{ll}
0 & 0 \\
a & b
\end{array}\right), a, b \in \mathbb{R}\right\}
$$




\begin{tabular}{|l|l|}
\hline $\mathbb{R} \bowtie \mathfrak{h}$ & $\mathfrak{g}$ \\
\hline \hline $\mathbb{R} \bowtie \mathbb{R}^{3}$ & $\mathbb{R}^{4}, \mathbb{R} \times \mathfrak{h}_{3}, \mathbb{R} \times \mathfrak{r}_{3}, \mathbb{R} \times \mathfrak{r}_{3, \lambda}, \mathbb{R} \times \mathfrak{r}_{3, \lambda}^{\prime}, \mathfrak{n}_{4}, \mathfrak{r}_{4}, \mathfrak{r}_{4, \lambda}, \mathfrak{r}_{4, \mu, \lambda}, \mathfrak{r}_{4, \mu, \lambda}^{\prime}$ \\
\hline $\mathbb{R} \bowtie \mathfrak{h}_{3}$ & $\mathbb{R} \times \mathfrak{h}_{3}, \mathfrak{n}_{4}, \mathfrak{r}_{4,0}, \mathfrak{d}_{4}, \mathfrak{d}_{4, \lambda}(\lambda \geqslant 1 / 2), \mathfrak{d}_{4, \lambda}^{\prime}(\lambda \geqslant 0), \mathfrak{h}_{4}$ \\
\hline $\mathbb{R} \bowtie \mathfrak{r}_{3}$ & $\mathbb{R} \times \mathfrak{r}_{3}, \mathfrak{r}_{4}, \mathfrak{r}_{4, \lambda},(\lambda \neq 0), \mathfrak{d}_{4,1}$ \\
\hline $\mathbb{R} \bowtie \mathfrak{r}_{3,0}$ & $\mathbb{R} \times \mathfrak{r}_{3, \lambda}, \mathfrak{a f f}(\mathbb{R}) \times \mathfrak{a f f}(\mathbb{R}), \mathfrak{r}_{4,0}, \mathfrak{d}_{4}, \mathfrak{d}_{4,1}$ \\
\hline $\mathbb{R} \bowtie \mathfrak{r}_{3,0}^{\prime}$ & $\mathbb{R} \times \mathfrak{r}_{3,0}^{\prime}, \mathfrak{a} \mathfrak{f} \mathfrak{f}(\mathbb{C})$ \\
\hline $\mathbb{R} \bowtie \mathfrak{r}_{3, \lambda}$ & $\mathbb{R} \times \mathfrak{r}_{3, \lambda}, \mathfrak{a f f}(\mathbb{R}) \times \mathfrak{a f f}(\mathbb{R})(\lambda=-1), \mathfrak{a f f}(\mathbb{C})(\lambda=1), \mathfrak{r}_{4, \lambda}, \mathfrak{r}_{4, \mu, \lambda}$, \\
& $\mathfrak{h}_{4}(\lambda=2), \mathfrak{d}_{4, \lambda}, \mathfrak{d}_{4,1-\lambda}$ \\
\hline $\mathbb{R} \bowtie \mathfrak{r}_{3, \lambda}^{\prime}$ & $\mathbb{R} \times \mathfrak{r}_{3, \lambda}^{\prime}, \mathfrak{a} \mathfrak{f} \mathfrak{f}(\mathbb{C}), \mathfrak{r}_{4, \mu, \lambda}^{\prime}$ \\
\hline
\end{tabular}

Table 3:

with respect to $\{z, w\}$. If $0 \rightarrow \mathfrak{a f f}(\mathbb{R}) \rightarrow \mathfrak{g} \rightarrow \mathfrak{g} / \mathfrak{a} \mathfrak{f} \mathfrak{f}(\mathbb{R}) \rightarrow 0$ splits, then there is a subalgebra $\mathfrak{h}$ of $\mathfrak{g}$ isomorphic to $\mathfrak{g} / \mathfrak{a} \mathfrak{f} \mathfrak{f}(\mathbb{R})$ such that $\mathfrak{g}=\mathfrak{h} \ltimes \mathfrak{a f f}(\mathbb{R})$. Set

$$
\rho: \mathfrak{h} \rightarrow \operatorname{Der} \mathfrak{a f f}(\mathbb{R}), \quad \rho(u)=\left.\operatorname{ad}(u)\right|_{\mathfrak{a f f}(\mathbb{R})}, \quad u \in \mathfrak{h} .
$$

Then $\rho$ is a Lie algebra homomorphism.

(i) In this case $\mathfrak{h}=\mathbb{R}^{2}$, so the image of $\rho$ is an abelian subalgebra of Der $\mathfrak{a f f}(\mathbb{R})$, hence it is one dimensional. Let $\mathbb{R}^{2}=\langle x, y\rangle$, aff $(\mathbb{R})=\langle z, w\rangle$. We may assume that $\rho(y)=0$. Let $\rho(x)=\left(\begin{array}{ll}0 & 0 \\ a & b\end{array}\right)$. If $a=b=0$ the assertion follows. If $b \neq 0$ we may assume that $b=1$ and we can reduce to $a=0$ by changing $z$ to $z$-aw. Hence, we may assume that the only non zero brackets are $[x, w]=w,[z, w]=w$ and therefore $\mathfrak{g}=\langle x-z, y\rangle \times\langle z, w\rangle$ where $\langle x-z, y\rangle \cong \mathbb{R}^{2}$. If $b=0, a \neq 0$, we may assume that $a=1$, therefore $\mathfrak{g}=\langle x+w, y\rangle \times\langle z, w\rangle$ where $\langle x+w, y\rangle \cong \mathbb{R}^{2}$ and the desired assertion follows.

(ii) We have $\mathfrak{h}=\mathfrak{a f f}(\mathbb{R})=\langle x, y\rangle,[x, y]=y$, and the following possibilities for $\rho$ :

$$
\rho(x)=\left(\begin{array}{ll}
0 & 0 \\
a & 1
\end{array}\right), \quad \rho(y)=\left(\begin{array}{ll}
0 & 0 \\
1 & 0
\end{array}\right) \quad \text { or } \quad \rho(x)=\left(\begin{array}{cc}
0 & 0 \\
a & b
\end{array}\right), \quad \rho(y)=0 .
$$

We show next that, in both cases, $\mathfrak{g} \cong \mathfrak{a f f}(\mathbb{R}) \times \mathfrak{a f f}(\mathbb{R})$.

If the first possibility occurs, take $\langle x-z+a w, y+w\rangle$ and $\langle z-a w, w\rangle$. These are complementary ideals isomorphic to $\mathfrak{a f f}(\mathbb{R})$, hence $\mathfrak{g} \cong \mathfrak{a} \mathfrak{f}(\mathbb{R}) \times \mathfrak{a} \mathfrak{f}(\mathbb{R})$.

In the second case, take $\langle x-b z+a w, y\rangle$ and $\langle z, w\rangle$, which are ideals isomorphic to $\mathfrak{a f f}(\mathbb{R})$, and the desired assertion follows.

\subsection{Product structures of type $\mathbb{R} \bowtie \mathfrak{h}$}

We exhibit in Table 3 realizations of the Lie algebras obtained in Theorem 1.5 as double Lie algebras where $\mathfrak{h}$ is a three dimensional subalgebra. Note that the problem of finding such a decomposition is equivalent to the determination of the three dimensional subalgebras. 
- $\mathbb{R} \bowtie \mathbb{R}^{3}$ : The Lie algebras $\mathbb{R} \times \mathfrak{h}_{3}, \mathbb{R} \times \mathfrak{r}_{3}, \mathbb{R} \times \mathfrak{r}_{3, \lambda}, \mathbb{R} \times \mathfrak{r}_{3, \lambda}^{\prime}, \mathfrak{n}_{4}, \mathfrak{r}_{4}, \mathfrak{r}_{4, \lambda}, \mathfrak{r}_{4, \mu, \lambda}$ and $\mathfrak{r}_{4, \mu, \lambda}^{\prime}$ were obtained in Theorem 1.5 as semidirect extensions of $\mathbb{R}^{3}$.

- $\mathbb{R} \bowtie \mathfrak{h}_{3}$ : The Lie algebras $\mathbb{R} \times \mathfrak{h}_{3}, \mathfrak{n}_{4}, \quad \mathfrak{d}_{4}, \quad \mathfrak{d}_{4, \lambda}(\lambda \geqslant 1 / 2), \quad \mathfrak{d}_{4, \lambda}^{\prime}(\lambda \geqslant 0)$, and $\mathfrak{h}_{4}$ were obtained in Theorem 1.5 as semidirect extensions of $\mathfrak{h}_{3}$. On the other hand,

$$
\mathfrak{r}_{4,0} \cong\left\langle e_{1}\right\rangle \bowtie\left\langle e_{0}, e_{2}, e_{3}\right\rangle
$$

- $\mathbb{R} \bowtie \mathfrak{r}_{3}:$

$$
\begin{aligned}
\mathfrak{r}_{4} & \cong\left\langle e_{3}\right\rangle \bowtie\left\langle e_{0}, e_{1}, e_{2}\right\rangle, \\
\mathfrak{r}_{4, \lambda} & \cong\left\langle e_{1}\right\rangle \bowtie\left\langle e_{0}, e_{2}, e_{3}\right\rangle, \quad \lambda \neq 0, \\
\mathfrak{d}_{4,1} & \cong\left\langle e_{0}\right\rangle \ltimes\left\langle e_{0}+e_{2}, e_{1}, e_{3}\right\rangle .
\end{aligned}
$$

- $\mathbb{R} \bowtie \mathfrak{r}_{3,0}$ :

$$
\begin{aligned}
\mathfrak{a f f}(\mathbb{R}) \times \mathfrak{a f f}(\mathbb{R}) & \cong\left\langle e_{0}\right\rangle \ltimes\left\langle e_{1}, e_{2}, e_{3}\right\rangle, \\
\mathfrak{r}_{4,0} & \cong\left\langle e_{3}\right\rangle \ltimes\left\langle e_{0}, e_{1}, e_{2}\right\rangle, \\
\mathfrak{d}_{4} & \cong\left\langle e_{2}\right\rangle \bowtie\left\langle e_{0}, e_{1}, e_{3}\right\rangle, \\
\mathfrak{d}_{4,1} & \cong\left\langle e_{1}\right\rangle \bowtie\left\langle e_{0}, e_{2}, e_{3}\right\rangle .
\end{aligned}
$$

- $\mathbb{R} \bowtie \mathfrak{r}_{3,0}^{\prime}: \mathfrak{a f f}(\mathbb{C}) \cong\left\langle e_{0}\right\rangle \ltimes\left\langle e_{1}, e_{2}, e_{3}\right\rangle$

- $\mathbb{R} \bowtie \mathfrak{r}_{3, \lambda}: \mathfrak{a} \mathfrak{a f}(\mathbb{R}) \times \mathfrak{a} \mathfrak{f} \mathfrak{f}(\mathbb{R})$ was obtained in Theorem 1.5 as a semidirect extension of $\mathfrak{r}_{3,-1}$.

$$
\begin{aligned}
\mathfrak{h}_{4} & =\left\langle e_{2}\right\rangle \bowtie\left\langle e_{0}, e_{1}, e_{3}\right\rangle \cong \mathbb{R} \bowtie \mathfrak{r}_{3,2}, \quad \lambda=2, \\
\mathfrak{a f f f}(\mathbb{C}) & =\left\langle e_{1}\right\rangle \bowtie\left\langle e_{0}, e_{2}, e_{3}\right\rangle \cong \mathbb{R} \ltimes \mathfrak{r}_{3,1}, \quad \lambda=1, \\
\mathfrak{r}_{4, \lambda} & \cong\left\langle e_{3}\right\rangle \bowtie\left\langle e_{0}, e_{1}, e_{2}\right\rangle, \\
\mathfrak{r}_{4, \mu, \lambda} & \cong\left\langle e_{2}\right\rangle \bowtie\left\langle e_{0}, e_{1}, e_{3}\right\rangle, \\
\mathfrak{r}_{4, \mu, \lambda} & \cong \mathbb{R} \bowtie \mathfrak{r}_{4, \mu}=\left\langle e_{3}\right\rangle \bowtie\left\langle e_{0}, e_{1}, e_{2}\right\rangle, \\
\mathfrak{d}_{4, \lambda} & \cong\left\langle e_{2}\right\rangle \bowtie\left\langle e_{0}, e_{1}, e_{3}\right\rangle, \mathfrak{d}_{4,1-\lambda} \cong\left\langle e_{1}\right\rangle \bowtie\left\langle e_{0}, e_{2}, e_{3}\right\rangle .
\end{aligned}
$$

- $\mathbb{R} \bowtie \mathfrak{r}_{3, \lambda}^{\prime}$ :

$$
\begin{aligned}
\mathfrak{a} \mathfrak{f} \mathfrak{f}(\mathbb{C}) & \cong\left\langle e_{0}\right\rangle \ltimes\left\langle\lambda e_{0}-e_{1}, e_{2}, e_{3}\right\rangle, \\
\mathfrak{r}_{4, \mu, \lambda}^{\prime} & \cong\left\langle e_{1}\right\rangle \bowtie\left\langle e_{0}, e_{2}, e_{3}\right\rangle .
\end{aligned}
$$

\section{Applications: Manin triples and complex product struc- tures}

\subsection{Manin triples on 4-dimensional solvable Lie algebras}

An important example of double Lie algebras are Manin triples $[\mathbf{L W}]$. We recall that a Manin triple is a double Lie algebra $\left(\mathfrak{g}, \mathfrak{g}_{+}, \mathfrak{g}_{-}\right)$with an invariant metric, that is, a non degenerate symmetric bilinear form $(,$,$) which satisfies:$

$$
([x, y], z)+(y,[x, z])=0 \quad \text { for all } x, y, z \in \mathfrak{g}
$$


such that $\mathfrak{g}_{+}$and $\mathfrak{g}_{-}$are isotropic subalgebras. In particular $\mathfrak{g}=\mathfrak{g}_{+} \bowtie \mathfrak{g}_{-}$, where $\mathfrak{g}_{+}$and $\mathfrak{g}_{-}$have the same dimension. Thus, Manin triples are special cases of paracomplex structures.

The next proposition makes use of $[\mathbf{B K}]$ and the results of the previous section to obtain that there is only one four dimensional solvable Lie algebra giving rise to Manin triples.

Proposition 3.1. Let $\left(\mathfrak{g}, \mathfrak{g}_{+}, \mathfrak{g}_{-}\right)$be a Manin triple such that $\mathfrak{g}$ is a non abelian four dimensional solvable Lie algebra. Then $\mathfrak{g}$ is isomorphic to $\mathfrak{d}_{4}$ with the invariant metric given by:

$$
\alpha=\left(e_{0}, e_{3}\right)=\left(e_{1}, e_{2}\right), \quad \alpha \neq 0,
$$

where the isotropic subalgebras $\mathfrak{g}_{+}$and $\mathfrak{g}_{-}$are given as follows:

(i) $\mathfrak{g}_{+}=\left\langle e_{0}+\mu e_{2}, e_{1}-\mu e_{3}\right\rangle, \quad \mathfrak{g}_{-}=\left\langle e_{0}+\nu e_{2}, e_{1}-\nu e_{3}\right\rangle$ with $\mu \neq \nu$; or

(ii) $\mathfrak{g}_{+}=\left\langle e_{0}+\mu e_{2}, e_{1}-\mu e_{3}\right\rangle, \quad \mathfrak{g}_{-}=\left\langle e_{2}, e_{3}\right\rangle: \mu, \nu \in \mathbb{R}$.

Proof. According to $[\mathbf{B K}]$ a non abelian solvable Lie algebra which admits an invariant metric is isomorphic either to $\mathfrak{d}_{4,0}^{\prime}$ or $\mathfrak{d}_{4}$. It was proved in Theorem 2.7 that $\mathfrak{d}_{4,0}^{\prime}$ does not admit paracomplex structures. Thus, we need to investigate the possible paracomplex structures on $\mathfrak{d}_{4}$. It is easy to see that the metric on $\mathfrak{d}_{4}$ given by:

$$
\left(e_{0}, e_{3}\right)=\left(e_{1}, e_{2}\right)=\alpha, \quad \text { with } \alpha \neq 0,
$$

is invariant. Any two-dimensional isotropic non abelian subalgebra of $\mathfrak{d}_{4}$ is isometrically isomorphic to:

$$
\left\langle e_{0}+\mu e_{2}, e_{1}-\mu e_{3}\right\rangle,
$$

where the isometric isomorphism is given by $\phi\left(e_{1}\right)=e_{2}, \phi\left(e_{i}\right)=-e_{i}, i=0,3$. On the other hand, any two-dimensional isotropic abelian subalgebra is isometrically isomorphic to:

$$
\left\langle e_{2}, e_{3}\right\rangle .
$$

It follows from 2.4 that $\mathfrak{d}_{4}$ does not admit a decomposition of type $\mathbb{R}^{2} \bowtie \mathbb{R}^{2}$. If both $\mathfrak{g}_{+}$and $\mathfrak{g}_{-}$are isomorphic to $\mathfrak{a f f}(\mathbb{R})$ then we are led to case (i). In case $\mathfrak{g}_{+} \cong \mathfrak{a} \mathfrak{f} \mathfrak{f}(\mathbb{R})$ and $\mathfrak{g}_{-} \cong \mathbb{R}^{2}$ we obtain case (ii), and the proposition follows.

\subsection{Complex product structures on four dimensional solvable Lie alge- bras}

In this subsection we determine all four dimensional solvable Lie algebras which admit a complex product structure (see Table 4), using the classification of complex structures on this class of Lie algebras given in $[\mathbf{S J}, \mathbf{O 1}]$ together with the results in $\S 2.2$. We give in this way an alternative proof of a result by Blazić and Vukmirović $([\mathbf{B V}])$, where complex product structures were referred to as para-hypercomplex structures.

We recall that a complex structure on a Lie algebra $\mathfrak{g}$ is an endomorphism $J$ : $\mathfrak{g} \rightarrow \mathfrak{g}$ such that $J^{2}=-\operatorname{Id}$ and

$$
J[x, y]=[J x, y]+[x, J y]+J[J x, J y]
$$




\begin{tabular}{|c|c|c|}
\hline Lie algebra & Complex structure & Paracomplex structure \\
\hline $\mathfrak{a f f}(\mathbb{R}) \times \mathfrak{a} \mathfrak{f}(\mathbb{R})$ & $J e_{0}=e_{3}, J e_{1}=e_{2}$ & $\left\langle e_{0}, e_{1}\right\rangle \ltimes\left\langle e_{2}, e_{3}\right\rangle$ \\
\hline $\mathbb{R} \times \mathfrak{h}_{3}$ & $J e_{0}=-e_{3}, J e_{1}=e_{2}$ & $\left\langle e_{0}, e_{1}\right\rangle \ltimes\left\langle e_{2}, e_{3}\right\rangle$ \\
\hline $\mathbb{R} \times \mathfrak{r}_{3,0}$ & $J e_{3}=e_{0}, J e_{1}=e_{2}$ & $\left\langle e_{1}, e_{3}\right\rangle \ltimes\left\langle e_{0}, e_{2}\right\rangle$ \\
\hline $\mathbb{R} \times \mathfrak{r}_{3,1}$ & $J e_{0}=e_{1}, J e_{2}=e_{3}$ & $\left\langle e_{1}, e_{3}\right\rangle \ltimes\left\langle e_{0}, e_{2}\right\rangle$ \\
\hline $\mathfrak{a f f}(\mathbb{C})$ & $J e_{0}=e_{2}, J e_{1}=e_{3}$ & $\left\langle e_{0}, e_{1}\right\rangle \ltimes\left\langle e_{2}, e_{3}\right\rangle$ \\
\hline $\mathfrak{r}_{4,1}$ & $J e_{0}=e_{3}, J e_{1}=e_{2}$ & $\left\langle e_{0}, e_{1}\right\rangle \ltimes\left\langle e_{2}, e_{3}\right\rangle$ \\
\hline $\mathfrak{r}_{4, \lambda, \lambda, \lambda \neq 0}$ & $J e_{0}=e_{1}, J e_{2}=e_{3}$ & $\left\langle e_{0}, e_{2}\right\rangle \ltimes\left\langle e_{1}, e_{3}\right\rangle$ \\
\hline $\mathfrak{r}_{4, \mu, 1}, \mu \neq 0, \pm 1$ & $J e_{0}=e_{2}, J e_{1}=e_{3}$ & $\left\langle e_{0}, e_{1}\right\rangle \ltimes\left\langle e_{2}, e_{3}\right\rangle$ \\
\hline $\mathfrak{r}_{4, \mu, \lambda}^{\prime}$ & $J e_{0}=e_{1}, J e_{2}=e_{3}$ & $\left\langle e_{0}, e_{1}\right\rangle \ltimes\left\langle e_{2}, e_{3}\right\rangle$ \\
\cline { 2 - 3 } & $J e_{0}=e_{1}, J e_{2}=-e_{3}$ & $\left\langle e_{0}, e_{1}\right\rangle \ltimes\left\langle e_{2}, e_{3}\right\rangle$ \\
\hline \multirow{2}{*}{$\mathfrak{d}_{4}$} & $J e_{0}=-e_{1}, J e_{2}=e_{3}$ & $\left\langle e_{0}, e_{1}\right\rangle \ltimes\left\langle e_{2}, e_{3}\right\rangle$ \\
\cline { 2 - 3 } & $J e_{0}=e_{3}-e_{1}, J e_{1}=e_{0}-e_{2}$, & $\left\langle e_{0}, e_{2}\right\rangle \ltimes\left\langle e_{1}, e_{3}\right\rangle$ \\
\hline $\mathfrak{d}_{4,1}$ & $J e_{2}=e_{3}$ & $\left\langle e_{0}, e_{2}\right\rangle \ltimes\left\langle e_{1}, e_{3}\right\rangle$ \\
\hline $\mathfrak{d}_{4,1 / 2}$ & $J e_{0}=e_{1}, J e_{2}=-e_{3}$ & $\left\langle e_{0}, e_{1}\right\rangle \ltimes\left\langle e_{2}, e_{3}\right\rangle$ \\
\cline { 2 - 3 } & $J e_{0}=e_{3}, J e_{1}=e_{2}$ & $\left\langle e_{0}, e_{1}\right\rangle \ltimes\left\langle e_{2}, e_{3}\right\rangle$ \\
\cline { 2 - 3 } & $J e_{0}=e_{3}, J e_{1}=-e_{2}$ & $\left\langle e_{0}, e_{2}\right\rangle \ltimes\left\langle e_{1}, e_{3}\right\rangle$ \\
\hline \multirow{2}{*}{$\mathfrak{h}_{4}$} & $J e_{0}=e_{1}, J e_{2}=-2 e_{3}$ & $\left\langle e_{0}, e_{1}\right\rangle \ltimes\left\langle e_{2}, e_{3}\right\rangle$ \\
\hline $\mathfrak{d}_{4, \lambda}, \lambda \neq 1,1 / 2$ & $J e_{0}=(1-\lambda) e_{2}, J e_{1}=e_{3}$ & $\left\langle e_{0}, e_{1}\right\rangle \bowtie\left\langle e_{2}, e_{3}\right\rangle$ \\
\hline & $J e_{0}=-\lambda e_{1}, J e_{2}=e_{3}$ & \\
\hline & $J e_{0}=4 e_{2}, J e_{1}=4 e_{3}$ & $\left.e_{1}, e_{3}\right\rangle$ \\
\hline
\end{tabular}

Table 4: Complex product structures

for all $x, y \in \mathfrak{g}$. A complex product structure on a Lie algebra $\mathfrak{g}$ is a pair $\{J, E\}$ where $J$ is a complex structure and $E$ is a product structure on $\mathfrak{g}$ such that $J E=-E J$. This is equivalent to having a splitting of $\mathfrak{g}$ as $\mathfrak{g}=\mathfrak{g}_{+} \oplus \mathfrak{g}_{-}$, where $\mathfrak{g}_{+}$and $\mathfrak{g}_{-}$are Lie subalgebras of $\mathfrak{g}$ such that $\mathfrak{g}_{-}=J \mathfrak{g}_{+}$. From this it follows that $E$ is, in fact, a paracomplex structure on $\mathfrak{g}$.

At this point we refer the reader to Table 4 .

\section{Remarks.}

a) The Lie algebras $\mathbb{R} \times \mathfrak{r}_{3, \lambda}^{\prime}$ admit complex structures (see $[\mathbf{S J}]$ ) and paracomplex structures (see Table 2). Nevertheless, they do not admit any complex product structure. To show this, we state the following result, which is proved in $[\mathbf{A D}]$ :

Proposition 3.2. Let $\{J, E\}$ be a complex product structure on the Lie algebra $\mathfrak{g}$ and let $\left(\mathfrak{g}, \mathfrak{g}_{+}, \mathfrak{g}_{-}\right)$be the associated double Lie algebra. Then the following assertions are equivalent: 
(i) $J$ is an abelian complex structure, i.e., $[J x, J y]=[x, y]$ for all $x, y \in \mathfrak{g}$.

(ii) The Lie subalgebras $\mathfrak{g}_{+}$and $\mathfrak{g}_{-}$are abelian;

(iii) $E$ is an abelian product structure, i.e., $[E x, E y]=-[x, y]$ for all $x, y \in \mathfrak{g}$.

It is known that the Lie algebra $\mathbb{R} \times \mathfrak{r}_{3, \lambda}^{\prime}$ does not admit any abelian complex structure (see $[\mathbf{S J}])$. However, from Lemma 2.5 this Lie algebra admits only abelian paracomplex structures and thus, from the previous proposition, there is no complex product structure on $\mathbb{R} \times \mathfrak{r}_{3, \lambda}^{\prime}$.

b) The Lie algebra $\mathfrak{a f f}(\mathbb{C})$ admits other complex structures, given by:

$$
J_{\alpha, \beta} e_{0}=\frac{\alpha}{\beta} e_{0}+\frac{\alpha^{2}+\beta^{2}}{\beta} e_{1}, \quad J_{\alpha, \beta} e_{2}=e_{3}
$$

with $\alpha \in \mathbb{R}, \beta \in \mathbb{R} \backslash\{0\}$. However, there is no paracomplex structure on $\mathfrak{a f f}(\mathbb{C})$ which anticommutes with $J_{\alpha, \beta}$. Let us show this last assertion. It is known from Proposition 2.6 that $\mathfrak{a} \mathfrak{f} \mathfrak{f}(\mathbb{C})$ does not admit decompositions of type $\mathfrak{a f f}(\mathbb{R}) \bowtie \mathfrak{a} \mathfrak{f}(\mathbb{R})$. Also, since the complex structure $J_{\alpha, \beta}$ is not abelian, any complex product structure on $\mathfrak{a f f}(\mathbb{C})$ induces a decomposition of type $\mathfrak{a} \mathfrak{f}(\mathbb{R}) \bowtie \mathbb{R}^{2}$. Let $\mathfrak{h}$ be a subalgebra of $\mathfrak{a} \mathfrak{f}(\mathbb{C})$ isomorphic to $\mathfrak{a} \mathfrak{f}(\mathbb{R})$; then $\mathfrak{h}$ has a basis $u=e_{0}+a_{2} e_{2}, v=b_{2} e_{2}+b_{3} e_{3}$. Then $J_{\alpha, \beta} u=\frac{\alpha}{\beta} e_{0}+\frac{\alpha^{2}+\beta^{2}}{\beta} e_{1}-a_{3} e_{2}+$ $a_{2} e_{3}, J_{\alpha, \beta} v=-b_{3} e_{2}+b_{2} e_{3}$. Then we must have $[J u, J v]=0$ and from this we obtain the system

$$
\left\{\begin{array}{l}
\frac{\alpha^{2}+\beta^{2}}{\beta} b_{2}+\frac{\alpha}{\beta} b_{3}=0 \\
\frac{\alpha}{\beta} b_{2}-\frac{\alpha^{2}+\beta^{2}}{\beta} b_{3}=0
\end{array}\right.
$$

It is easy to see that the only solution of this system is $b_{2}=b_{3}=0$, i.e., $v=0$, a contradiction. Therefore, there are no product structures on $\mathfrak{a f f}(\mathbb{C})$ which anticommute with $J_{\alpha, \beta}$.

c) The Lie algebras $\mathfrak{d}_{4, \lambda}^{\prime}$ admit complex structures (see [O1]) but, according to Theorem 2.7, they do not admit any paracomplex structure. Hence, they do not carry complex product structures.

d) Table 4 shows examples of complex product structures on $\mathbb{R} \times \mathfrak{h}_{3}$ and $\mathfrak{r}_{4,1,1}$. On the other hand, all equivalence classes of complex product structures on these Lie algebras were determined in $[\mathbf{A S}]$, section 6.2.

\section{Appendix I - Matrix realizations}

We exhibit below matrix realizations of the indecomposable Lie algebras listed in Theorem 1.5, where indecomposable means that they do not split as a direct product of lower dimensional Lie algebras. All matrices have real coefficients. 


$$
\begin{aligned}
& \mathfrak{n}_{4}: \quad\left(\begin{array}{cccc}
0 & x & 0 & w \\
0 & 0 & x & y \\
0 & 0 & 0 & z \\
0 & 0 & 0 & 0
\end{array}\right) \\
& \mathfrak{a f f}(\mathbb{C}): \quad\left(\begin{array}{ccc}
x & z & y \\
-z & x & w \\
0 & 0 & 0
\end{array}\right) \\
& \mathfrak{r}_{4}: \quad\left(\begin{array}{cccc}
x & x & 0 & y \\
0 & x & x & z \\
0 & 0 & x & w \\
0 & 0 & 0 & 0
\end{array}\right) \\
& \mathfrak{r}_{4, \lambda}: \quad\left(\begin{array}{cccc}
x & 0 & 0 & y \\
0 & \lambda x & x & z \\
0 & 0 & \lambda x & w \\
0 & 0 & 0 & 0
\end{array}\right) \\
& \begin{array}{r}
\mathfrak{r}_{4, \mu, \lambda}: \\
\mu \lambda \neq 0,-1<\mu \leqslant \lambda \leqslant 1 \\
\text { or }-1=\mu \leqslant \lambda<0
\end{array} \quad\left(\begin{array}{cccc}
x & 0 & 0 & y \\
0 & \mu x & 0 & z \\
0 & 0 & \lambda x & w \\
0 & 0 & 0 & 0
\end{array}\right)
\end{aligned}
$$

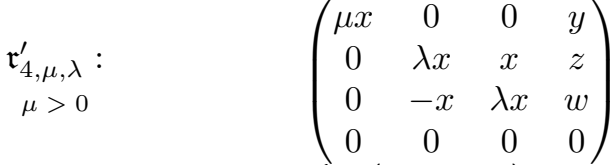

$$
\begin{aligned}
& \mathfrak{d}_{4}: \quad\left\{\begin{array}{lll}
0 & x & z \\
0 & w & y \\
0 & 0 & 0
\end{array}\right) \\
& \mathfrak{d}_{4, \lambda}: \\
& \lambda \geqslant \frac{1}{2} \\
& \left(\begin{array}{cccc}
w & 0 & 0 & x \\
0 & -w & 0 & y \\
-\frac{1}{2} y & \frac{1}{2} x & 0 & z \\
0 & 0 & 0 & 0
\end{array}\right) \\
& \left(\begin{array}{ccc}
w & x & z \\
0 & (1-\lambda) w & y \\
0 & 0 & 0
\end{array}\right) \\
& \left(\begin{array}{cccc}
\lambda w & 0 & 0 & x \\
0 & (1-\lambda) w & 0 & y \\
-\frac{1}{2} y & \frac{1}{2} x & w & z \\
0 & 0 & 0 & 0
\end{array}\right) \\
& \mathfrak{d}_{4, \lambda}^{\prime}: \quad\left(\begin{array}{cccc}
\lambda w & w & 0 & x \\
-w & \lambda w & 0 & y \\
-\frac{1}{2} y & \frac{1}{2} x & 2 \lambda w & z \\
0 & 0 & 0 & 0
\end{array}\right) \\
& \mathfrak{h}_{4}: \quad\left(\begin{array}{cccc}
\frac{1}{2} w & w & 0 & x \\
0 & \frac{1}{2} w & 0 & y \\
-\frac{1}{2} y & \frac{1}{2} x & w & z \\
0 & 0 & 0 & 0
\end{array}\right)
\end{aligned}
$$




\section{Appendix II - Comparison with previous classifications}

In this section we carry out a comparison with various results which can be found in the literature. Our main goal is to establish a correspondence between the description obtained by other authors and the Lie algebras appearing in Theorem 1.5.

\section{3.}

We start by comparing our results with the ones obtained by Dozias as appearing in $[\mathbf{V e}]$, Table 1.1, p. 180.

\begin{tabular}{|c|c|c|c|c|c|c|c|c|}
\hline $\mathfrak{g}_{4,1}$ & $\mathfrak{g}_{4,2}$ & $\mathfrak{g}_{4,3}$ & $\mathfrak{g}_{4,4}$ & $\mathfrak{g}_{4,5}(\alpha, \beta)$ & $\mathfrak{g}_{4,6}(\alpha)$ & $\mathfrak{g}_{4,7}$ & $\mathfrak{g}_{4,8}(\alpha, \beta)$ & $\mathfrak{g}_{4,9}(0)$ \\
\hline $\mathfrak{d}_{4,0}$ & $\mathfrak{a} \mathfrak{f} \mathfrak{f}(\mathbb{C})$ & $\mathfrak{n}_{4}$ & $\mathfrak{r}_{4,0}$ & $\mathfrak{r}_{4, \alpha, \beta}$ & $\mathfrak{r}_{4, \alpha}$ & $\mathfrak{r}_{4}$ & $\mathfrak{r}_{4, \alpha, \beta}^{\prime}$ & $\mathfrak{d}_{4}$ \\
\hline
\end{tabular}

\begin{tabular}{|c|c|c|}
\hline $\mathfrak{g}_{4,9}(\alpha), \alpha \neq 0$ & $\mathfrak{g}_{4,10}$ & $\mathfrak{g}_{4,11}(\alpha)$ \\
\hline $\mathfrak{d}_{4,1-1 / \alpha}$ & $\mathfrak{h}_{4}$ & $\mathfrak{d}_{4, \alpha}^{\prime}$ \\
\hline
\end{tabular}

\section{4 .}

We recall below the classification given by Mubarakzyanov $[\mathbf{M u}]$ and then we establish the correspondence with the algebras appearing in Theorem 1.5.

\begin{tabular}{|c|llll|}
\hline $\begin{array}{c}\text { Notation } \\
\text { in }[\mathbf{M u}]\end{array}$ & \multicolumn{4}{|c|}{ Lie bracket relations } \\
\hline $\mathfrak{g}_{4,1}$ & {$\left[e_{2}, e_{4}\right]=e_{1}$} & {$\left[e_{3}, e_{4}\right]=e_{2}$} & \\
$\mathfrak{g}_{4,2}$ & {$\left[e_{1}, e_{4}\right]=\alpha e_{1}$} & {$\left[e_{2}, e_{4}\right]=e_{2}$} & {$\left[e_{3}, e_{4}\right]=e_{2}+e_{3}$} & \\
$\mathfrak{g}_{4,3}$ & {$\left[e_{1}, e_{4}\right]=e_{1}$} & {$\left[e_{3}, e_{4}\right]=e_{2}$} & & \\
$\mathfrak{g}_{4,4}$ & {$\left[e_{1}, e_{4}\right]=e_{1}$} & {$\left[e_{2}, e_{4}\right]=e_{1}+e_{2}$} & {$\left[e_{3}, e_{4}\right]=e_{2}+e_{3}$} & \\
$\mathfrak{g}_{4,5}$ & {$\left[e_{1}, e_{4}\right]=e_{1}$} & {$\left[e_{2}, e_{4}\right]=\beta e_{2}$} & {$\left[e_{3}, e_{4}\right]=\gamma e_{3}$} & $-1 \leqslant \gamma \leqslant \beta \leqslant 1$, \\
& & & $\gamma \beta \neq 0$ \\
$\mathfrak{g}_{4,6}$ & {$\left[e_{1}, e_{4}\right]=\alpha e_{1}$} & {$\left[e_{2}, e_{4}\right]=p e_{2}-e_{3}$} & {$\left[e_{3}, e_{4}\right]=e_{2}+p e_{3}$} & $\alpha \neq 0, \mathrm{p} \geqslant 0$ \\
$\mathfrak{g}_{4,7}$ & {$\left[e_{2}, e_{3}\right]=e_{1}$} & {$\left[e_{1}, e_{4}\right]=2 e_{1}$} & {$\left[e_{2}, e_{4}\right]=e_{2}$} & {$\left[e_{3}, e_{4}\right]=e_{2}+e_{3}$} \\
$\mathfrak{g}_{4,8}$ & {$\left[e_{2}, e_{3}\right]=e_{1}$} & {$\left[e_{1}, e_{4}\right]=(1+h) e_{1}$} & {$\left[e_{2}, e_{4}\right]=e_{2}$} & {$\left[e_{3}, e_{4}\right]=h e_{3}$,} \\
& & & $|\mathrm{h}| \leqslant 1$ \\
$\mathfrak{g}_{4,9}$ & {$\left[e_{2}, e_{3}\right]=e_{1}$} & {$\left[e_{1}, e_{4}\right]=2 p e_{1}$} & {$\left[e_{2}, e_{4}\right]=p e_{2}-e_{3}$} & {$\left[e_{3}, e_{4}\right]=e_{2}+p e_{3}$,} \\
& & & & $\mathrm{p} \geqslant 0$ \\
$\mathfrak{g}_{4,10}$ & {$\left[e_{1}, e_{3}\right]=e_{1}$} & {$\left[e_{2}, e_{3}\right]=e_{2}$} & {$\left[e_{1}, e_{4}\right]=-e_{2}$} & {$\left[e_{2}, e_{4}\right]=e_{1}+e_{3}$} \\
\hline
\end{tabular}

The correspondence is as follows:

\begin{tabular}{|c|c|c|c|c|c|c|c|c|c|}
\hline $\mathfrak{g}_{4,1}$ & $\mathfrak{g}_{4,2}$ & $\mathfrak{g}_{4,3}$ & $\mathfrak{g}_{4,4}$ & $\mathfrak{g}_{4,5}$ & $\mathfrak{g}_{4,6}$ & $\mathfrak{g}_{4,7}$ & $\mathfrak{g}_{4,8}$ & $\mathfrak{g}_{4,9}$ & $\mathfrak{g}_{4,10}$ \\
\hline $\mathfrak{n}_{4}$ & $\mathfrak{r}_{4, \alpha}$ & $\mathfrak{r}_{4,0}$ & $\mathfrak{r}_{4}$ & $\mathfrak{r}_{4, \beta, \gamma}$ & $\mathfrak{r}_{4, \alpha, p}^{\prime}$ & $\mathfrak{h}_{4}$ & $\mathfrak{d}_{4}, \mathfrak{d}_{4,1 / 1+b}$ & $\mathfrak{d}_{4, a}^{\prime}$ & $\mathfrak{a} \mathfrak{f} \mathfrak{f}(\mathbb{C})$ \\
\hline
\end{tabular}




\section{5 .}

In $[\mathbf{P S W Z}]$ invariants of real Lie algebras of dimension at most five are given. In particular, a list of four dimensional solvable Lie algebras, based on that of $[\mathbf{M u}]$, is shown in Table I, p. 988. The relation with Theorem 1.5 is :

\begin{tabular}{|c|c|c|c|c|c|c|c|}
\hline$A_{4,1}$ & $A_{4,2}{ }^{a}$ & $A_{4,3}$ & $A_{4,4}$ & $A_{4,5}{ }^{a, b}$ & $A_{4,6}{ }^{a, b}$ & $A_{4,7}$ & $A_{4,8}$ \\
\hline $\mathfrak{n}_{4}$ & $\mathfrak{r}_{4, a}$ & $\mathfrak{r}_{4,0}$ & $\mathfrak{r}_{4}$ & $\mathfrak{r}_{4, a, b}$ & $\mathfrak{r}_{4, a, b}^{\prime}$ & $\mathfrak{h}_{4}$ & $\mathfrak{d}_{4}$ \\
\hline
\end{tabular}

\begin{tabular}{|c|c|c|c|}
\hline$A_{4,9}{ }^{b}$ & $A_{4,10}$ & $A_{4,11}{ }^{a}$ & $A_{4,12}$ \\
\hline $\mathfrak{d}_{4,1 / 1+b}$ & $\mathfrak{d}_{4,0}^{\prime}$ & $\mathfrak{d}_{4, a}^{\prime}$ & $\mathfrak{a f f}(\mathbb{C})$ \\
\hline
\end{tabular}

3.6.

The classification of complex structures on four dimensional Lie algebras was carried out by Snow in $[\mathbf{S J}]$ and by Ovando in $[\mathbf{O 1}]$. To achieve this classification a description is given in $[\mathbf{S J}]$, p. 400, of four dimensional solvable Lie algebras when the commutator ideal has dimension 1 or 2 . We compare below the list given by Snow with the one obtained in Theorem 1.5.

\begin{tabular}{|c|c|c|c|c|c|}
\hline$S 1$ & $S 2$ & $S 3$ & $S 4$ & $S 5_{d}, d \neq 0$ & $S 6$ \\
\hline $\mathbb{R} \times \mathfrak{h}_{3}$ & $\mathbb{R}^{2} \times \mathfrak{a f f}(\mathbb{R})$ & $\mathfrak{r}_{4,0}$ & $\mathfrak{n}_{4}$ & $\mathbb{R} \times \mathfrak{r}_{3, d}$ & $\mathbb{R} \times \mathfrak{r}_{3}$ \\
\hline
\end{tabular}

\begin{tabular}{|c|c|c|c|}
\hline$S 7_{0, c}, \quad c>0$ & $S 7_{1, c}, 4 c>1$ & $S 8$ & $S 9$ \\
\hline $\mathbb{R} \times \mathfrak{r}_{3,0}^{\prime}$ & $\mathbb{R} \times \mathfrak{r}_{3, \sqrt{4 c-1}}^{\prime}$ & $\mathfrak{a f f}(\mathbb{R}) \times \mathfrak{a f f}(\mathbb{R})$ & $\mathfrak{d}_{4,1}$ \\
\hline
\end{tabular}

\begin{tabular}{|c|c|c|}
\hline$S 10_{d, d}, d \neq 0$ & $S 10_{d, c}, \quad c \neq d, d \neq 0$ & $S 11_{d, c}, d^{2}-4 c<0, d=0,1$ \\
\hline $\mathbb{R} \times \mathfrak{r}_{3, d}$ & $\mathfrak{a f f}(\mathbb{R}) \times \mathfrak{a} \mathfrak{f} \mathfrak{f}(\mathbb{R})$ & $\mathfrak{a f f}(\mathbb{C})$ \\
\hline
\end{tabular}

The above correspondence shows that some of the families appearing in $[\mathbf{S J}]$ become a single Lie algebra. Also, there exist isomorphisms between different families. We give below the proof of these statements.

- S7

We recall from $[\mathbf{S J}]$ the definition of the Lie algebra $S 7_{d, c}, d^{2}-4 c<0, d=0$ or 1 with basis $x, y, z, w$ :

$$
[x, y]=w, \quad[x, w]=-c y+d w .
$$

Observe that if $d=0$ then $c>0$ and $\operatorname{ad}(x)_{\mid \mathfrak{g}^{\prime}}$ has eigenvalues $\pm i c$. We can take a real basis of $\mathfrak{g}^{\prime}$ such that $\operatorname{ad}(x)_{\mid \mathfrak{g}^{\prime}}$ takes the form $\left(\begin{array}{cc}0 & c \\ -c & 0\end{array}\right)$. Changing $x$ by $x / c$ we see that $S 7_{0, c} \cong \mathbb{R} \times \mathfrak{e}(2)$ for all $c>0$. If $c=1$, then $\operatorname{ad}(x)_{\mid \mathfrak{g}^{\prime}}$ has eigenvalues $1 / 2 \pm i \lambda / 2$, where $\lambda=\sqrt{4 c-1}$. Taking $x^{\prime}=x / 2$, there exists a real basis of $\mathfrak{g}^{\prime}$ such that $\operatorname{ad}\left(x^{\prime}\right)_{\mid \mathfrak{g}^{\prime}}$ takes the form $\left(\begin{array}{cc}1 & \lambda \\ -\lambda & 1\end{array}\right)$, hence $S 7_{1, c} \cong \mathbb{R} \times \mathfrak{r}_{3, \lambda}^{\prime}$ for all $c$ such that $1-4 c<0$. 


\section{- $\mathrm{S} 10$}

Consider next the Lie algebra $S 10_{d, c}, \quad c, d \in \mathbb{R}, d \neq 0$ :

$$
[x, y]=y, \quad[x, w]=d w, \quad[z, y]=y, \quad[z, w]=c w .
$$

If $c=d$, then changing $z$ by $x-z$, we see that $S 10_{d, d} \cong \mathbb{R} \times \mathfrak{r}_{3, d}$ for all $d \neq 0$. If $c \neq d$, let $x^{\prime}, y^{\prime}, z^{\prime}, w^{\prime}$ be the basis of $S 10_{1,0}$ satisfying (15) and $x, y, z, w$ the corresponding basis of $S 10_{d, c}$. Define a linear map $\psi: S 10_{d, c} \rightarrow S 10_{1,0}$ by

$\psi(x)=x^{\prime}+(d-1) z^{\prime}, \quad \psi(y)=w^{\prime}, \quad \psi(z)=x^{\prime}+(c-1) z^{\prime}, \quad \psi(w)=y^{\prime}$.

It turns out that $\psi$ is a Lie algebra isomorphism for all $c \neq d$ and therefore $S 10_{d, c} \cong S 10_{1,0} \cong \mathfrak{a f f}(\mathbb{R}) \times \mathfrak{a f f}(\mathbb{R})$, where the last isomorphism follows by changing $x^{\prime}$ to $x^{\prime}-z^{\prime}$.

- S11

Consider the Lie algebra $S 11_{d, c}, d^{2}-4 c<0, d=0,1$ :

$[x, y]=y, \quad[x, w]=w, \quad[z, y]=w, \quad[z, w]=-c y+d w$.

If $d=0$, then $\operatorname{ad}(z / c)_{\mid \mathfrak{g}^{\prime}}$ has eigenvalues $\pm i$ and there exists a real basis of $\mathfrak{g}^{\prime}$ such that $\operatorname{ad}(z / c)=\left(\begin{array}{rr}0 & 1 \\ -1 & 0\end{array}\right)$, hence $S 11_{0, c} \cong \mathfrak{a f f}(\mathbb{C})$ for all $c>0$.

If $d=1$, then $\operatorname{ad}(z)_{\mid \mathfrak{g}^{\prime}}$ has eigenvalues $1 / 2 \pm i \lambda / 2$, where $\lambda=\sqrt{4 c-1}$. Taking $z^{\prime}=z / 2$, there exists a real basis of $\mathfrak{g}^{\prime}$ such that $\operatorname{ad}\left(z^{\prime}\right)_{\mid \mathfrak{g}^{\prime}}$ takes the form $\left(\begin{array}{cc}1 & \lambda \\ -\lambda & 1\end{array}\right)$. Changing $z^{\prime}$ to $z^{\prime \prime}=\left(z^{\prime}-x\right) / \lambda$, so that $\operatorname{ad}\left(z^{\prime \prime}\right)=\left(\begin{array}{cc}0 & 1 \\ -1 & 0\end{array}\right)$, we conclude that $S 11_{1, c} \cong \mathfrak{a} \mathfrak{f f}(\mathbb{C})$ for all $c$ such that $4 c>1$.

Finally, in case the commutator ideal is three dimensional, we establish the correspondence with Table 1 in [O1], p. 22.

\begin{tabular}{|c|c|c|c|}
\hline$A 1_{\lambda_{1}, \lambda_{2}}, \quad \lambda_{1} \neq \lambda_{2} \in \mathbb{R} \backslash\{0,1\}$ & $A 1_{\lambda, \bar{\lambda}}, \operatorname{Im} \lambda \neq 0$ & $A 2_{\lambda}, \lambda \in \mathbb{R} \backslash\{0,1\}$ & $A 3_{\lambda}, \lambda \in \mathbb{R} \backslash\{0,1\}$ \\
\hline $\mathfrak{r}_{4, \lambda_{1}, \lambda_{2}}$ & $\mathfrak{r}_{4,1 / \operatorname{Im} \lambda, \operatorname{Re} \lambda / \operatorname{Im} \lambda}^{\prime}$ & $\mathfrak{r}_{4, \lambda, \lambda}$ & $\mathfrak{r}_{4, \lambda}$ \\
\hline
\end{tabular}

\begin{tabular}{|c|c|c|c|c|c|c|c|c|}
\hline$A 4$ & $A 5$ & $A 6$ & $H 1$ & $H 2$ & $H 3$ & $H 4$ & $H 5_{\lambda}, \quad \lambda \in \mathbb{R} \backslash\{0,1\}$ & $H 6_{\lambda}, \quad \lambda \in \mathbb{C} \backslash \mathbb{R}$ \\
\hline $\mathfrak{r}_{4,1,1}$ & $\mathfrak{r}_{4,1}$ & $\mathfrak{r}_{4}$ & $\mathfrak{d}_{4}$ & $\mathfrak{d}_{4,0}^{\prime}$ & $\mathfrak{d}_{4,1 / 2}$ & $\mathfrak{h}_{4}$ & $\mathfrak{d}_{4, \lambda}$ & $\mathfrak{d}_{4,-1 / \operatorname{Im} \lambda}^{\prime}$ \\
\hline
\end{tabular}




\section{Appendix III - Some known results related to 4-dimensional geometry}

Using the characterization of homogeneous manifolds of negative curvature given by Heintze in $[\mathbf{H}]$ we can conclude that the following four dimensional Lie algebras do admit metrics with negative curvature:

- $\mathfrak{r}_{4, \mu, \lambda}, \quad 0<\mu \leqslant \lambda \leqslant 1$,

- $\mathfrak{r}_{4, \mu, \lambda}^{\prime}, \mu>0, \lambda>0$,

- $\mathfrak{d}_{4, \lambda}, 1 / 2 \leqslant \lambda<1$,

- $\mathfrak{d}_{4, \lambda}^{\prime}, \quad \lambda>0$,

- $\mathfrak{h}_{4}$.

Concerning non positive sectional curvature, we can mention a result appearing in $[$ Dru], where it is proved that a left invariant metric with non positive curvature on a four dimensional solvable Lie group either has geometric rank one or it comes from an inner product on $\mathfrak{a f f}(\mathbb{R}) \times \mathfrak{a f f}(\mathbb{R})$ or $\mathbb{R} \times \mathfrak{r}_{3,1}$, up to scaling.

We understand that the classification of rank one four dimensional homogeneous spaces of non positive curvature is not known. On the other hand, Jensen classified in $[\mathbf{J}]$ the four dimensional Lie algebras admitting Einstein metrics:

- $\mathbb{R} \times \mathfrak{r}_{3,1}$,

- $\mathfrak{r}_{4,1,1}$,

- $\mathbb{R} \times \mathfrak{r}_{3,0}^{\prime}$,

- $\mathfrak{r}_{4, \lambda, \lambda}^{\prime}, \lambda>0$.

- $\mathfrak{d}_{4, \lambda}, \quad \lambda \geqslant 1 / 2$,

Among these, it follows from [Al] that there are only two four dimensional Lie algebras admitting Einstein metrics of non positive curvature: $\mathfrak{r}_{4,1,1}$ and $\mathfrak{d}_{4,1 / 2}$. Concerning left invariant anti-self-dual metrics on four dimensional Lie groups, it was proved in [DS] (Theorem 1.6) that if a four dimensional Lie group admits such a metric, then its Lie algebra is one of the following:

- $\mathfrak{d}_{4,1 / 2}$,

- $\mathfrak{d}_{4, \lambda}^{\prime}, \lambda>0$.

It is proved in $[\mathbf{F 2}]$ that $\mathfrak{d}_{4,2}$ is the only four dimensional solvable Lie algebra admitting an almost Kähler structure whose Ricci tensor is invariant with respect to the almost complex structure.

The classification of complex structures on four dimensional solvable Lie algebras was carried out by Snow in $[\mathbf{S J}]$, when the commutator subalgebra has dimension one or two, and by Ovando in $[\mathbf{O} \mathbf{1}]$, when the commutator subalgebra is three dimensional. The classification of hypercomplex structures was obtained in [B1].

Concerning the existence of symplectic structures, it is shown in $[\mathbf{F G}]$ that the solvmanifold obtained as a quotient of $E(1,1)$, the simply connected Lie group with Lie algebra $\mathfrak{e}(1,1)$, by a lattice, admits a symplectic structure but no complex structure. The classification of symplectic structures on four dimensional Lie algebras is done in [02], where the cohomology of all four dimensional solvable Lie algebras is computed. 
The hyper-Kähler metrics conformal to left invariant metrics metric on four dimensional Lie groups were determined in [B2]. It turns out that the solvable Lie groups appearing in this list are those with Lie algebra $\mathbb{R}^{4}, \mathfrak{a f f}(\mathbb{C}), \mathfrak{r}_{4,1,1}$ or $\mathfrak{d}_{4,1 / 2}$. It was proved in $[\mathbf{F 1}]$ that the cotangent bundle of a Lie group with Lie algebra $\mathfrak{a} \mathfrak{f}(\mathbb{C})$ or $\mathfrak{r}_{4,1,1}$ also admits a metric conformal to a hyper-Kähler metric.

The determination of hypersymplectic structures on four dimensional Lie algebras was carried out in $[\mathbf{A n}]$. According to this, the only Lie algebras admitting such a structure are $\mathbb{R}^{4}, \mathbb{R} \times \mathfrak{h}_{3}, \mathfrak{r}_{4,-1,-1}$ and $\mathfrak{d}_{4,2}$.

\section{References}

[Al] D. Alekseevsky, Homogeneous Riemannian spaces of negative curvature, Math. USSR Sbornik 25(1) (1975), 87-109.

[An] A. Andrada, Hypersymplectic 4-dimensional Lie algebras, preprint. Available at arXiv:math.DG/0310462

[AD] A. Andrada, I. Dotti, Double products and hypersymplectic structures on $\mathbb{R}^{4 n}$, preprint. Available at arXiv:math.DG/0401294

[AS] A. Andrada, S. Salamon, Complex product structures on Lie algebras, to appear in Forum Math.

[ACFM] L. C. de Andrés, L. Cordero, M. Fernández, J. J. Mencía, Examples of four-dimensional compact locally conformal Kähler solvmanifolds, Geom. Dedicata 29, No.2, 227-232 (1989).

[AFGM] L. C. de Andrés, M. Fernández, A. Gray, J.Mencía, Moduli spaces of complex structures on compact four dimensional nilmanifolds, Boll. Unione Mat. Ital., VII Ser., A 5, No.3, 381-389 (1991).

[B1] M. L. Barberis, Hypercomplex structures on four dimensional Lie groups, Proc. Amer. Math. Soc. 125 (1997), 1043-1054.

[B2] M.L. Barberis, Hyper-Kähler metrics conformal to left invariant metrics on four dimensional Lie groups, Math. Phys., Analysis and Geometry 6 (1), (2003), 1-8.

[BK] H. Baum, I. Kath, Doubly extended Lie groups - Curvature, holonomy and Parallel spinors, Differential Geometry and its Applications 19(2003), 253-280.

[BV] N. Blazić, S. Vukmirović, Four-dimensional Lie algebras with a parahypercomplex structure, available at arXiv: math.DG/0310180

[CFG] V. Cruceanu, P. Fortuny and P.M. Gadea, A survey on paracomplex geometry, Rocky Mountain J. Math. 26(1) (1996), 83-115. 
[DS] V. De Smedt, S. Salamon, Anti-self dual metrics on Lie groups, Contemp. Math. 308 (2002), 63-75.

[D] J. Dozias, Sur les algèbres de Lie résolubles réelles de dimension inférieure ou égale à 5 , Thèse de 3 cycle, novembre 1963, Faculté des Sciences de Paris.

[Dru] M.J. Druetta, Visibility and rank one in homogeneous spaces of $K \leqslant 0$, Trans. AMS 304 (1987), 307-321.

[FG] M. Fernández and A. Gray, Compact symplectic solvmanifolds not admitting complex structures, Geom. Dedic. 34 (1990), 295-299.

[F1] A. Fino, Cotangent bundles of 4-dimensional hypercomplex Lie groups, Manuscr. Math. 109(4) (2002), 527-541.

[F2] A. Fino, Almost Kähler 4-dimensional Lie groups with J-invariant Ricci tensor, preprint math.DG/0308014.

[GOV] V. Gorbatsevich, A. Onishchik, E. Vinberg, Structure of Lie groups and Lie algebras, English transl. in Encycl. Math Sc. 41, Springer-Verlag, Berlin, Heidelberg, 1994.

[H] E. Heintze, On homogeneous manifolds of negative curvature, Math. Ann, 211 (1974), 23-34.

[J] G.R. Jensen, Homogeneous Einstein metrics of dimension four, J. Differential Geom. 3 (3) (1969), 309-349.

[LM] M. de León and D. Martín de Diego, Almost product structures in mechanics, Diff. Geom. Appl., Proc. Conf., Aug. 28 - Sept. 1, 1995, Brno, Czech Republic, Masaryk University, Brno 1996, 539-548.

[LW] Lu, J.-H. and Weinstein, A.: Poisson Lie groups, dressing transformations and Bruhat decompositions. J. Diff. Geom. 31 (1990), 501-526.

[Mi] J. Milnor, Curvatures of left invariant metrics on Lie groups, Advances in Mathematics, 21 (1976), 293-329.

[Miq] V. Miquel, Some examples of Riemannian almost-product manifolds, Pac. J. Math. 111 (1984), 163-178.

[Mu G.M. Mubarakzyanov, On solvable Lie algebras, Izv. Vyssh. Uchebn. Zaved., Mat 32 (1) (1963), 114-123 (Russian). Zbl 166,42.

[N] A.M. Naveira, A classification of Riemannian almost product manifolds, Rendiconti di Matematica (3) 1983 Vol. 3, Serie VII, 577-592.

[01] G. Ovando, Invariant complex structures on solvable real Lie groups, Manuscripta Math., 103, (2000), 19-30.

[O2] G. Ovando, Complex, symplectic and Kähler structures on four dimensional Lie groups, to appear in Rev. Un. Mat. Argentina. 
[PSWZ] J. Patera, R.T. Sharp, P. Winternitz, H. Zassenhaus, Invariants of real low dimension Lie algebras, J. Math. Physics, 17, (1976), 986-994.

[P] P. Petravchuk, Lie algebras decomposable as a sum of an abelian and a nilpotent subalgebra, Ukr. Math. J. 40(3) (1988), 385-388.

[SJ] J. E. Snow, Invariant complex structures on four dimensional solvable real Lie groups, Manuscripta Math, 66 (1990), pag 397-412.

[Ve] M. Vergne, Construction de représentations irréductibles des groupes de Lie résolubles, in $\mathrm{P}$. Bernat et al., Représentations des groupes de Lie résolubles, Monographies de la Société Mathématique de France 4, Dunod, Paris, 1972.

This article may be accessed via WWW at http://www.rmi.acnet.ge/hha/ or by anonymous ftp at

ftp://ftp.rmi.acnet.ge/pub/hha/volumes/2005/n1a2/v7n1a2.(dvi,ps,pdf)

A. Andrada andrada@mate.uncor.edu

M. L. Barberis barberis@mate.uncor.edu

I. G. Dotti idotti@mate.uncor.edu

G. P. Ovando ovando@mate.uncor.edu

CIEM, FaMAF,

Universidad Nacional de Córdoba, Ciudad Universitaria,

(5000) Córdoba,

Argentina 